\title{
The Most Pathogenic Transthyretin Variant, L55P, Forms Amyloid Fibrils under Acidic Conditions and Protofilaments under Physiological Conditions ${ }^{\dagger}$
}

\author{
Hilal A. Lashuel, ${ }^{\ddagger}$ Christine Wurth, ${ }^{\S}$ Linda Woo, ${ }^{\ddagger}$ and Jeffery W. Kelly*, \\ Department of Chemistry and The Skaggs Institute of Chemical Biology, The Scripps Research Institute, 10550 North Torrey \\ Pines Rd, MB 12, La Jolla, California 92037, and Department of Pharmacy, Swiss Federal Institute of Technology (ETH), \\ Winterhurerstr. 190, CH 8057 Zurich, Switzerland
}

Received May 4, 1999; Revised Manuscript Received August 3, 1999

\begin{abstract}
The L55P transthyretin (TTR) familial amyloid polyneuropathy-associated variant is distinct from the other TTR variants studied to date and the wild-type protein in that the L55P tetramer can dissociate to the monomeric amyloidogenic intermediate and form fibril precursors under physiological conditions ( $\mathrm{pH} 7.0,37^{\circ} \mathrm{C}$ ). The activation barrier associated with L55P-TTR tetramer dissociation is lower than the barrier for wild-type transthyretin dissociation, which does not form fibrils under physiological conditions. The L55P-TTR tetramer is also very sensitive to acidic conditions, readily dissociating to form the monomeric amyloidogenic intermediate between $\mathrm{pH}$ 5.5-5.0 where the wildtype TTR adopts a nonamyloidogenic tetrameric structure. The formation of the L55P monomeric amyloidogenic intermediate involves subtle tertiary structural changes within the $\beta$-sheet rich subunit as discerned from Trp fluorescence, circular dichroism analysis, and ANS binding studies. The assembly of the L55P-TTR amyloidogenic intermediate at physiological $\mathrm{pH}(\mathrm{pH} 7.5)$ affords protofilaments that elongate with time. TEM studies suggest that the entropic barrier associated with filament assembly (amyloid fibril formation) is high in vitro, amyloid being defined by the laterally assembled four filament structure observed by Blake upon isolation of "fibrils" from the eye of a FAP patient. The L55P-TTR protofilaments formed in vitro bind Congo red and thioflavin $\mathrm{T}$ (albeit more weakly than the fibrils produced at acidic $\mathrm{pH}$ ), suggesting that the structure observed probably represents an amyloid precursor. The structural continuum from misfolded monomer through protofilaments, filaments, and ultimately fibrils must be considered as a possible source of pathology associated with these diseases.
\end{abstract}

There are several human neurodegenerative diseases putatively caused by extracellular protein deposition (1). The most familiar of these include the amyloid diseases, e.g. Alzheimer's disease and senile systemic amyloidosis (27). In all amyloid diseases, proteins undergo conformational changes either before or coincident with their self-assembly into highly ordered amyloid fibrils. Amyloid fibrils having a cross $\beta$-sheet quaternary structure are typically deposited in the brain and/or in peripheral tissues and have been linked to neurodegeneration and/or organ dysfunction by genetic evidence $(2,8-11)$. The amyloid hypothesis implicates amyloid fibrils as the causative agent in amyloid diseases through a gain of function phenotype. $(1,7,9,12-14)$. Recent results from several laboratories, including our own, demonstrate the existence of preamyloid quaternary structural intermediates (protofilaments), implying that the whole process of amyloid fibril formation must be considered as a

\footnotetext{
The authors gratefully acknowledge primary financial support from the National Institute of Health (R01 DK46335) and secondary support from The Skaggs Institute of Chemical Biology, The Lita Annenberg Hazen Foundation and The American Chemical Society (Organic Division Fellowship to HAL).

Department of Chemistry and the Skaggs Institute of Chemical Biology.

$\S$ Department of Pharmacy.

* To whom correspondence should be addressed. Telephone: 619784-9601; Fax: 619-784-9610; E-mail: jkelly@scripps.edu.
}

possible cause of pathology, including the first step in the process, which is protein misfolding in the case of structurally well-defined amyloid proteins (14-21).

Understanding the mechanism of how a folded protein is converted into an insoluble amyloid fibril is critical for developing inhibition strategies against amyloid formation $(1,6,21-25)$. Small molecule inhibitors may prevent amyloid disease and, at the very least, provide a means to critically test the amyloid hypothesis, particularly if they inhibit the misfolding process $(23-26)$. There are currently 17 amyloidogenic proteins that self-assemble and appear to cause amyloid disease (1). These proteins do not appear to be functionally related. A comparison of these proteins, based on sequence or tertiary structure, reveals a lack of clear homology. Nevertheless, these amyloidogenic proteins are capable of forming highly ordered amyloid fibrils of similar structure in vivo as discerned from X-ray fibril diffraction, electron and atomic force microscopy morphology, and the ability to bind amyloid selective dyes, such as Congo red and thioflavin $\mathrm{T}(27,28)$. The ability of structurally and

\footnotetext{
${ }^{1}$ Abbreviations: TTR, transthyretin; FAP, familial amyloid polyneuropathy; SSA, senile systemic amyloidosis; RBP, retinol binding protein; SDS-PAGE, sodium dodecyl sulfate-polyacrylamide gel electrophoresis; OD, optical density; Z 3-14, N-tetradecyl-N, N-dimethyl-3-ammonio-1-propane-sulfonate; ANS, 1-anilino-8-naphthalenesulfonic acid; CD, circular dichroism; EM, electron microscopy; $\mathrm{s}$, sedimentation coefficient; S, Svedberg unit $\left(10^{-13} \mathrm{sec}\right)$.
} 
functionally diverse amyloidogenic proteins to form amyloid fibrils having a common structure can be explained if these proteins adopt an alternative $\beta$-sheet rich fold as a result of partial denaturation, in the case of folded proteins or linked self-assembly and conformational changes in the case of unfolded peptides such as $\mathrm{A} \beta$. The altered conformation allows a subset of human proteins to self-assemble into a cross- $\beta$ amyloid structure, perhaps through common structural intermediates $(1,6,16,21,29-32)$.

Transthyretin (TTR) is one of several structurally welldefined amyloidogenic proteins (including lysozyme and immunoglobulin light chain) that undergoes a conformational change and self-associates into amyloid fibrils as a result of a partial denaturing environment, a destabilizing mutation, or both $(1,33-37)$. Extensive structural and genetic information on transthyretin make it an ideal amyloid protein to probe the biochemical mechanism of fibril formation and to understand the basis for pathology $(1,2,29,30,33,38-$ 40 ), especially when considering that it is not necessary to cross the blood brain barrier to inhibit TTR fibril formation with a small molecule. The process of amyloid fibril formation is implicated as the causative agent in two types of TTR-associated amyloid diseases, familial amyloid polyneuropathy (FAP), which affects approximately 1 in 100,000 persons, and senile systemic amyloidosis (SSA), which affects, to some extent, $25 \%$ of the population over 80 years of age. The amyloid fibrils of SSA patients are composed exclusively of wild-type transthyretin (WT-TTR) $(4,41)$. In contrast, one of more than seventy single-site variants of TTR is the predominant component of the FAP fibrils (typically $>90 \%)(2,42,43)$. Familial amyloid polyneuropathy refers to an early onset disease where the pathology ranges from peripheral neuropathy to organ dysfunction $(2,8,42,44-$ 46). While the presence of an amyloidogenic mutation does not always lead to FAP, all of the early-onset FAP cases are associated with a TTR mutation, suggesting that the mutation is necessary but not sufficient for disease onset $(2,40)$. The structures of approximately ten FAP-associated variants have been solved by X-ray crystallography, revealing folds that are nearly identical with WT- TTR (47).

Extensive data support the hypothesis that single-site FAPassociated mutations function by destabilizing the native protein fold, favoring dissociation and the formation of an alternatively folded monomeric amyloidogenic intermediate under conditions where the wild-type tetramer is stable. The formation of the amyloidogenic intermediate allows selfassembly and, ultimately, amyloid fibril formation $(1,6,16$, $21,29,30,48)$. A strong correlation exists between the instability of the FAP variants in vitro $(\mathrm{pH}$ or chaotropeinduced) and the age of FAP onset in vivo, with the least stable variant (L55P-TTR) having the earliest onset and being the most pathogenic (onset during the second decade, death by 38 years of age) $(16,29,30)$.

The L55P mutation is in the D- $\beta$-strand on the outer edge of the TTR tetramer, Figure 1. Introduction of Pro into this strand should disrupt its structure as verified by the recent $\mathrm{X}$-ray crystal structure; however, this disruption is localized and does not prevent tetramer formation (49). Previous studies suggest that the C-strand-loop-D-strand region of TTR becomes a disordered loop upon formation of the amyloidogenic intermediate $(1,6,16,21,50-52)$. It is known that multiple mutations and deletions in the D-strand

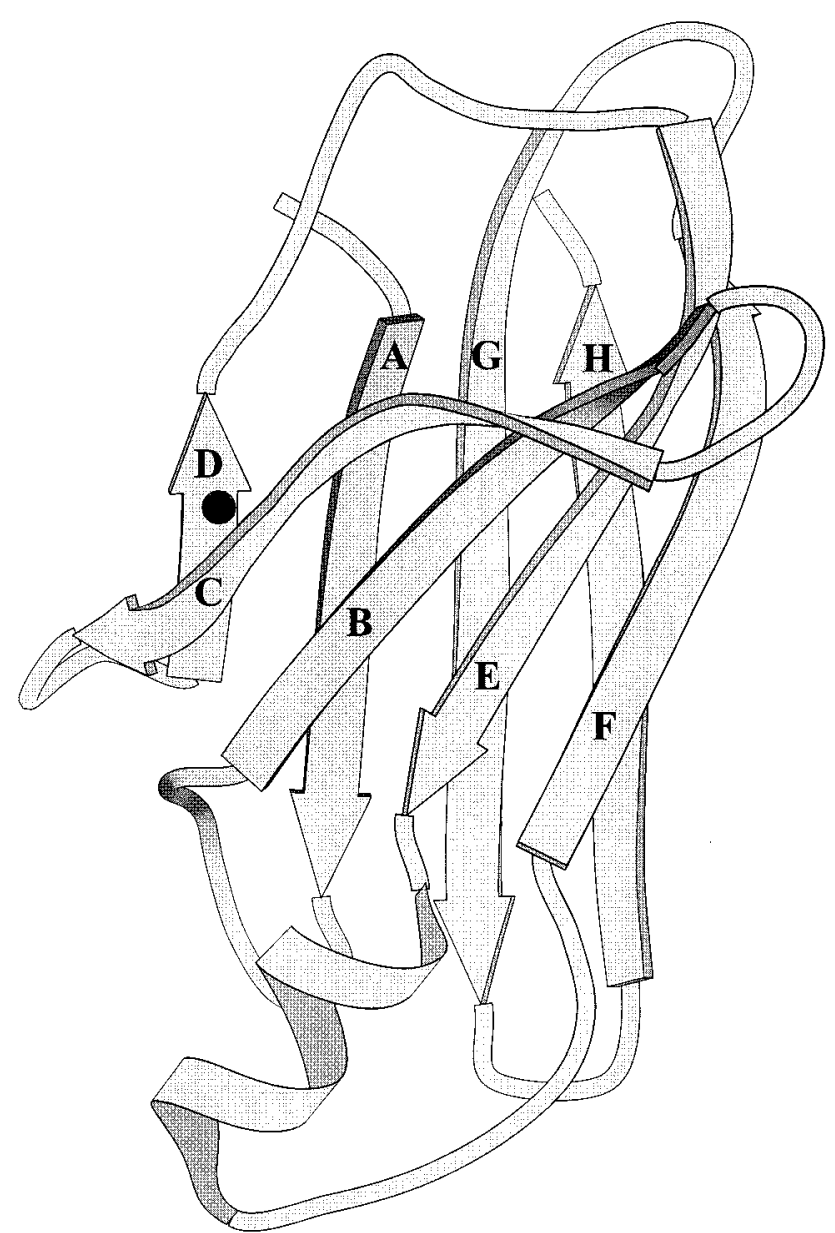

FIGURE 1: Ribbon diagram of the wild-type TTR monomer generated from the crystal structural coordinates of the subunit in the tetramer (38) using the program Molscript (69). Several of the FAP causing mutations are found in the C-strand loop D-strand region including the most lethal mutation $\mathrm{L} 55 \mathrm{P}$ which is represented by the black sphere in the D-strand.

(residues 53-55) result in significant destabilization of the TTR fold and enhanced amyloid fibril formation under physiological conditions, corroborating the involvement of this region in the formation of the TTR amyloidogenic intermediate (53). The L55P-TTR crystallography results seem to confirm the structural basis for the extreme amyloidogenicity, in that L55P-TTR is already partially biased toward the putative $\mathrm{C}$-strand-loop-D-strand rearranged structure, characteristic of the amyloidogenic intermediate (49). Unlike WT-TTR, the L55P variant can be converted into protofilaments via partial thermal denaturation $\left(37^{\circ} \mathrm{C}\right)$ under physiological conditions (vide infra) (16). Sedimentation velocity experiments, reveal that the L55P-TTR tetramer slowly dissociates to a monomer $\left(\mathrm{pH} 7.0,37^{\circ} \mathrm{C}\right)$ prior to protofilament formation. Prior to this discovery, little attention was paid to the amyloidogenic potential of L55P-TTR under physiological conditions, hence the presence of soluble amyloid protofilaments went undetected in solutions that were thought to be homogeneous (tetrameric). These oligomeric intermediates in older samples complicate the interpretation of biophysical measurements.

Motivated by the fact that L55P-TTR has not been wellcharacterized in solution, we sought to understand the basis for its extreme amyloidogenicity. Fluorescence and circular dichroism spectroscopy were used to monitor tertiary struc- 
tural changes, while electron microscopy and sedimentation velocity studies were employed to characterize self-assembly mediated by acid and thermally induced partial denaturation. The results demonstrate that L55P-TTR $\left(0.2 \mathrm{mg} / \mathrm{mL}, 37^{\circ} \mathrm{C}\right.$, $\mathrm{pH}$ 7.0) slowly dissociates to the alternatively folded amyloidogenic monomer that self-assembles into protofilaments under physiological conditions, a process which is dependent on concentration, $\mathrm{pH}$, and temperature. Importantly, studies on L55P-TTR amyloidogencity suggest a common mechanism for TTR amyloid fibril formation and, therefore, a common therapeutic strategy can be envisioned for preventing fibril formation in the diseases SSA and FAP $(1,16)$, even though L55P-TTR is drastically more amyloidogenic than WT-TTR.

\section{MATERIALS AND METHODS}

The silver staining kit used to visualize SDS-PAGE gels was purchased from Pierce or Biorad. The detergent Z 3-14 was obtained from Calbiochem. The Congo red used in this study was procured from Sigma and was recrystallized from water/ethanol as described previously (31). All other reagents used were of the highest quality available from Fisher, Sigma, or Calbiochem and were used without further purification.

Expression and Purification of L55P $\left(\mathrm{Met}^{-1}\right)$-TTR. The L55P $\left(\mathrm{Met}^{-1}\right)$-TTR variant was isolated and purified from recombinant sources following the procedures described below and developed by David Wemmer's group (UC Berkeley) (54). The L55P ( $\left.\mathrm{Met}^{-1}\right)$-TTR pMMHa plasmid was transformed into competent BL21 DE3 Epicurian Gold cells (Strategene). Two 1.5 L flasks of LB medium with ampicillin $(100 \mu \mathrm{g} / \mathrm{mL})$ were innoculated with fresh $5 \mathrm{~mL}$ overnight cultures. Cells were grown at $37^{\circ} \mathrm{C}$ with vigorous shaking until they reached an $\mathrm{OD}_{600 \mathrm{~nm}}$ of $0.6(\mathrm{AU})$; they were then induced with $2 \mathrm{mM}$ IPTG. After $19 \mathrm{~h}$ of post-induction agitation, the cells were harvested, resuspended in $25 \mathrm{mM}$ Tris buffer (pH 7.5, 2 mM EDTA, 0.1\% (v/v) Triton X-100), and lysed by two cycles of freeze-thaw, followed by sonication $(4 \times 30 \mathrm{~s})$. After removal of the cell debris by centrifugation, L55P $\left(\mathrm{Met}^{-1}\right)$-TTR was ammonium sulfate precipitated $(50-90 \%)$. The pellet was resuspended in a

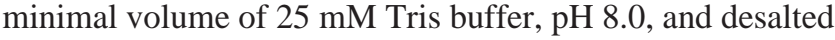
over a Hi-Prep desalting column (Pharmacia). The desalted protein solution was heated at $60^{\circ} \mathrm{C}$ for $30 \mathrm{~min}$ in order to precipitate out contaminating proteins. The supernatant was loaded onto a Source Q column (Pharmacia) and eluted using a linear $\mathrm{NaCl}$ gradient $(0.20-0.35 \mathrm{M} \mathrm{NaCl})$ employing 25 $\mathrm{mM}$ Tris buffer, $\mathrm{pH} 8.0$, affording $180 \mathrm{mg}$ of $>95 \%$ pure TTR/3.0 L of liquid culture. An SDS-PAGE gel was utilized to assess protein purity. Because of its tendency to aggregate upon storage for more than two weeks (even at $4{ }^{\circ} \mathrm{C}$ ), purified L55P-TTR was always ammonium sulfate precipitated $(90 \%)$ and stored as a pellet at $4{ }^{\circ} \mathrm{C}$. Dissolution of the pellet and use of the sample within a few days yields tetrameric L55P-TTR, according to ultracentrifugation analysis (see below). To circumvent any complications caused by the presence of soluble aggregates in L55P-TTR stock solutions stored for more than a week, analytical ultracentrifugation methods were routinely employed with aged samples to make sure that experiments commenced with a hydrodynamically defined pure tetrameric protein (see below). We have found that centrifugation of stock solutions at $50000 \mathrm{rpm}$ (237000g, preparative Beckman centrifuge) followed by filtration through $0.2 \mu \mathrm{m}$ membranes is sufficient to remove the vast majority of soluble aggregates in aged L55P-TTR solutions.

Evaluating the Extent of L55P-TTR Amyloid Fibril Formation. The extent of L55P-TTR amyloid fibril formation mediated either by acid or thermal denaturation was evaluated by turbidity or optical density (OD) measurements at $330 \mathrm{~nm}$ in a standard UV cell $(55,56)$. In addition, a Congo red binding assay was utilized to quantify the amount of amyloid fibrils formed $(31,57)$. Dissolution of an ammonium sulfate pellet of L55P-TTR (10 mM phosphate buffer, $\mathrm{pH}$ 7.5, $100 \mathrm{mM} \mathrm{KCl}, 1 \mathrm{mM}$ EDTA), desalting over a Hi-Prep column (Pharmacia), and concentration of the sample results in a stock solution $(3-5 \mathrm{mg} / \mathrm{mL})$. This solution was diluted into $50 \mathrm{mM}$ sodium acetate or sodium phosphate buffer (100 $\mathrm{mM} \mathrm{KCl}$ ) at the desired $\mathrm{pH}$ in a glass test tube to evaluate the extent of amyloid fibril formation observed (final TTR concentration $=0.2 \mathrm{mg} / \mathrm{mL}$ ). The resulting stagnant solutions were incubated for 24 or $48 \mathrm{~h}$ at $37^{\circ} \mathrm{C}$. Prior to turbidity evaluation $\left(\mathrm{OD}_{330 \mathrm{~nm}}\right)$ the samples were vortexed to equally distribute the fibril suspension. To provide an independent measurement of the amount of fibrils formed, $50 \mu \mathrm{L}$ of the L55P-TTR suspension was added to $1150 \mu \mathrm{L}$ of a $10 \mu \mathrm{M}$ Congo red solution $(5 \mathrm{mM}$ phosphate, $150 \mathrm{mM} \mathrm{NaCl}, \mathrm{pH}$ 7.5) and the absorbances of the suspension at 477 and 540 $\mathrm{nm}$ were recorded. The quantity of fibrils formed was determined by the amount of Congo red bound to the amyloid fibrils (characterized by a red-shifted absorbance) in solution ( $\mathrm{mol}$ of Congo red bound $/ \mathrm{L}$ of amyloid suspension $=\mathrm{A}_{540 \mathrm{~nm}} /$ $25295-\mathrm{A}_{477 \mathrm{~nm}} / 46$ 306) (57, 58).

Probing the Time Course of Amyloid Fibril Formation via Turbidity. The time course of amyloid fibril formation was evaluated by monitoring the change in turbidity at $330 \mathrm{~nm}$ as a function of time and $\mathrm{pH}\left(25^{\circ} \mathrm{C}\right)$. Samples were prepared by adding $120 \mu \mathrm{L}$ of a L55P-TTR stock solution $(5 \mathrm{mg} / \mathrm{mL})$ to $2.88 \mathrm{~mL}$ of $50 \mathrm{mM}$ acetate buffer $(100 \mathrm{mM} \mathrm{KCl})$ at the desired $\mathrm{pH}$ in a standard UV cuvette to afford a L55P-TTR solution $(0.2 \mathrm{mg} / \mathrm{mL})$. The L55P-TTR solutions were placed immediately into a Hewlett-Packard (Palo Alto, CA) UV spectrophotometer (10 s dead time), and data collection was initiated thereafter by monitoring the change in OD at 330 $\mathrm{nm}$ employing mechanical stirring.

Probing the Stability of the Tetrameric Leu-55-Pro Structure via SDS-PAGE. The quaternary structural changes exhibited by L55P-TTR during acid denaturation were probed using an SDS-PAGE method developed in our laboratory $(29,31)$. SDS-PAGE analysis provides a fast and reliable means for evaluating the amount of tetramer present as it runs as a dimer on the SDS-PAGE gel (confirmed by analytical ultracentrifuge studies), provided TTR is not boiled in the SDS sample buffer (boiling results in the conversion of the dimer band to a monomer band) $(31,33)$. However, the SDS-PAGE method does not faithfully represent the amount of monomer present due to the fact that soluble aggregated forms of TTR dissociate into monomers in nonboiled SDS-PAGE buffer $(16,33)$. Although dimeric TTR could exist as a transient species, techniques such as sedimentation velocity/equilibrium analysis and analytical gel filtration, capable of resolving the TTR monomer, dimer, and tetramer, do not detect dimeric TTR.

The L55P-TTR samples $(0.2,0.1,0.01 \mathrm{mg} / \mathrm{mL})$ were incubated at $25^{\circ} \mathrm{C}$ in $50 \mathrm{mM}$ phosphate or acetate buffer to 
establish a monomer-tetramer equilibrium at the desired $\mathrm{pH}$. After incubation of the samples for 24 or $44 \mathrm{~h}$, the detergent Z 3-14 (from a $25 \mathrm{mg} / \mathrm{mL}$ stock Z 3-14 solution in water) was added to each TTR solution (the zwitterionic detergent $\mathrm{Z}$ 3-14 is used to prevent the refolding/reassociation of monomeric TTR to the native tetramer during the neutralization process necessary for gel loading) (31). Twenty $\mu \mathrm{L}$ of $0.6 \mathrm{M}$ phosphate buffer ( $\mathrm{pH} 7$ ) containing $0.5 \mathrm{mg} / \mathrm{mL} \mathrm{Z} \mathrm{3-14}$ was added to TTR solutions to neutralize the $\mathrm{pH}$. These samples $(20 \mu \mathrm{L})$ were mixed with $10 \mu \mathrm{L}$ of $5 \%$ SDS sample buffer in an Eppendorf tube and loaded without boiling onto a $12 \%$ SDS-PAGE gel that was run and stained using Gelcode Blue Staining Reagent (Pierce) or visualized by silver stain (Biorad; for $0.01 \mathrm{mg} / \mathrm{mL}$ samples). The SDSPAGE gels were analyzed by a Molecular Dynamics model (D1PC) computing densitometer and the band intensity data were plotted versus $\mathrm{pH}$ to depict the ratio of L55P-TTR tetramer to all other quaternary structural forms displayed as monomer by SDS-PAGE analysis. The midpoint $\left(\mathrm{pH}_{\mathrm{m}}\right)$ of the acid mediated tetramer to monomer/oligomer transition is the $\mathrm{pH}$ at which half of the TTR is in the tetrameric form by weight.

Evaluating Amyloidogenic L55P-TTR Quaternary Structural Changes via Analytical Ultracentrifugation. Sedimentation velocity experiments were utilized to assess sample homogeneity/heterogeneity and the distribution of quaternary structural species formed during acid and thermally induced denaturation/amyloid fibril formation. Concentrated TTR stock solutions $(5 \mathrm{mg} / \mathrm{mL}, 10 \mathrm{mM}$ phosphate, $100 \mathrm{mM} \mathrm{KCl}$, $1 \mathrm{mM}$ EDTA) were spun on a desktop centrifuge (16000g) for $15 \mathrm{~min}$ at $4{ }^{\circ} \mathrm{C}$ and all buffers were filtered through a $0.22 \mu \mathrm{m}$ filter before they were subjected to incubation. Transthyretin solutions $(0.2$ or $0.05 \mathrm{mg} / \mathrm{mL})$ were prepared by dilution of the centrifuged TTR stock solution into a filtered buffer (50 mM phosphate or acetate buffer, $100 \mathrm{mM}$ $\mathrm{KCl})$ at the desired $\mathrm{pH}$ and temperature $\left(4,25\right.$, or $\left.37^{\circ} \mathrm{C}\right)$.

The sedimentation properties of TTR as a function of $\mathrm{pH}$, temperature, concentration, and incubation time were obtained from velocity data collected on a temperaturecontrolled Beckman XL-I analytical ultracentrifuge (equipped with a An60Ti rotor and photoelectric scanner). A double sector cell, equipped with a $12 \mathrm{~mm}$ Epon centerpiece and quartz windows, was loaded with $400-420 \mu \mathrm{L}$ of sample using a blunt-end microsyringe. Data were collected at rotor speeds of 3000-60 $000 \mathrm{rpm}$ in continuous mode at 4 or 20 ${ }^{\circ} \mathrm{C}$, with a step size of $0.005 \mathrm{~cm}$ employing an average of 1-4 scans per point. The data were subjected to analysis using the Svedberg direct boundary fitting approach (for samples containing up to 3 components) to allow for $M_{\mathrm{W}}$ determination using the sedimentation coefficient and diffusion coefficient obtained from the appropriate fitting model (59). For samples containing a heterogeneous mixture of quaternary structural species, the van Holde and Weischet direct fitting and the time derivative (dc/dt) methods were used to analyze the distribution of species in solution represented by a range of $s$ values $(16,60-62)$.

Sedimentation Equilibrium Experiments. Sedimentation equilibrium experiments allow the quaternary structural changes required for amyloid fibril formation to be studied provided that the heterogeneity is limited to two species or, alternatively, four associating species that are in equilibrium. A TTR sample $(150 \mathrm{~mL})$ in a double sector cell with a charcoal-filled Epon centerpiece and sapphire windows at the desired $\mathrm{pH}$ and temperature was analyzed using rotor speeds from 3000-17 $000 \mathrm{rpm}$. All scans were performed at $280 \mathrm{~nm}(230 \mathrm{~nm}$ at lower concentration), with a step size of $0.001 \mathrm{~cm}$, employing 25 averaged scans. Samples were allowed to equilibrate for $24 \mathrm{~h}$, and duplicate scans $3 \mathrm{~h}$ apart were overlaid to determine that equilibrium had been reached. The data were analyzed by nonlinear least-squares analysis in the Origin software provided by Beckman (Palo Alto, CA) as described in detail previously (16).

Evaluating L55P-TTR pH-Dependent 2' and 3' Structural Changes by Far- and Near-UV Circular Dichroism. Circular dichroism (CD) spectroscopy was used to further evaluate the secondary and tertiary structural requirements for L55PTTR amyloid fibril formation (acid-induced). The CD spectra of L55P-TTR as a function of $\mathrm{pH}$ were recorded on an Aviv Model SF202 spectrometer $\left(25^{\circ} \mathrm{C}\right)$. Transthyretin solutions at $0.01 \mathrm{mg} / \mathrm{mL}$ (a concentration where aggregation is not a problem) at the desired $\mathrm{pH}(50 \mathrm{mM}$ acetate or phosphate buffer, $100 \mathrm{mM} \mathrm{KCl}$ ) were prepared by dilution of a $5 \mathrm{mg}$ / $\mathrm{mL}$ stock solution $(10 \mathrm{mM}$ phosphate, $100 \mathrm{mM} \mathrm{KCl}$, and 1 $\mathrm{mM}$ EDTA). CD studies carried out at $0.01 \mathrm{mg} / \mathrm{mL}$ were performed using $0.2 \mathrm{~cm}$ quartz cell for far- $(195-250 \mathrm{~nm})$ and a $10 \mathrm{~cm}$ quartz cell for near-UV CD (250-320). A step size of $0.2 \mathrm{~nm}$, an averaging time of $3 \mathrm{~s}$ and an average of 15 scans were recorded to generate the data reported in units of mean residue ellipticity. The far- and near-UV CD data were smoothed using a Stineman function (KalidaGraph software), which reduced the noise without perturbing the appearance of the data.

Evaluating the Tertiary Structural Changes Exhibited by L55P-TTR during Acid Denaturation via Fluorescence Spectroscopy. The tertiary structural changes occurring during L55P-TTR acid-induced denaturation/amyloid fibril formation were evaluated by monitoring the changes in the intrinsic tryptophan fluorescence of L55P-TTR as a function of $\mathrm{pH}$ at a concentration where self-assembly is not competitive. The L55P-TTR samples $(0.01 \mathrm{mg} / \mathrm{mL})$ were made by adding $10 \mu \mathrm{L}$ of a L55P-TTR stock solution $(1 \mathrm{mg} / \mathrm{mL})$ to the desired buffer solution $(990 \mu \mathrm{L}$ of $50 \mathrm{mM}$ acetate or phosphate, $100 \mathrm{mM} \mathrm{KCl}$ ). Fluorescence spectra of L55PTTR as a function of $\mathrm{pH}$ were recorded on Aviv model ATF 105 spectrofluorometer at $25^{\circ} \mathrm{C}$ in a $1 \mathrm{~cm}$ quartz cell. The excitation wavelength was set at $295 \mathrm{~nm}$ (slit width of 4 $\mathrm{nm}$ ) and the emission wavelength monitored at $340 \mathrm{~nm}$ (slit width of $8 \mathrm{~nm}$ ) to measure the tryptophan fluorescence exclusively.

ANS Binding as a Probe of Denaturation. ANS binding studies were employed to evaluate the relative hydrophobicity of the amyloidogenic intermediate formed under acidic partial denaturing conditions. A stock ANS solution (5.6 mM) was prepared, and its concentration determined by absorbance at $351 \mathrm{~nm}\left(\epsilon=6240 \mathrm{M}^{-1} \mathrm{~cm}^{-1}\right)$. To measure the binding of ANS to L55P-TTR as a function of $\mathrm{pH}, 3 \mathrm{~mL}$ of a $0.01 \mathrm{mg} /$ $\mathrm{mL}$ TTR solution in the appropriate buffer was prepared and equilibrated at $25^{\circ} \mathrm{C}$ for $20-28 \mathrm{~h}$. An aliquot of an ANS stock solution was then added to afford a final ANS concentration of $48 \mu \mathrm{M}$. The resulting solutions were further incubated at $25{ }^{\circ} \mathrm{C}$ for another $24 \mathrm{~h}$ prior to recording the fluorescence spectra. The fluorescence intensities were measured via excitation at $410 \mathrm{~nm}$, recording emission at $490 \mathrm{~nm}$. 
Probing the Assembly of the L55P-TTR Amyloidogenic Intermediate by Electron Microscopy and Dye Binding. Electron microscopy was utilized to evaluate the nature and structure of the L55P-TTR aggregates formed as a function of $\mathrm{pH}$, temperature, and ionic strength. Imaging was enabled by placing $5 \mu \mathrm{L}$ of the sample on a carbon-coated copper grid, allowing the solution to stand for 2 min before removing excess solution. The grid was then washed once with distilled water and once with $1 \%$ uranyl acetate before staining the sample with fresh $1 \%$ uranyl acetate for another 2 min. Excess uranyl acetate was blotted with Whatman filter paper. The samples were then evaluated using a Phillips CM-100 $(100 \mathrm{kV})$ electron microscope. The grid was thoroughly examined to obtain an overall sense of the structures present in the sample. The protofilaments formed at $\mathrm{pH} 7.5$ were evaluated by thioflavin $\mathrm{T}$ and Congo red binding. For thioflavin $\mathrm{T}$ binding studies, $100 \mu \mathrm{L}$ of $0.2 \mathrm{mg} / \mathrm{mL}$ L55PTTR solutions, containing protofilaments, were added to 500 $\mu \mathrm{L}$ of a $10 \mu \mathrm{M}$ thioflavin T solution in PBS buffer ( $\mathrm{pH} 6.6$ ). The fluorescence intensities were measured via excitation at $450 \mathrm{~nm}$ and the emission spectra was recorded from 460 to $630 \mathrm{~nm}$. Congo red binding analysis was performed as described previously (29).

\section{RESULTS}

Evaluating the Extent of L55P-TTR Acid DenaturationAmyloid Fibril Formation. The extent of L55P-TTR amyloid fibril formation (protofilament and filament formation) as a function of $\mathrm{pH}\left(37^{\circ} \mathrm{C}\right)$ was evaluated by monitoring turbidity $(330 \mathrm{~nm})$ and quantitative Congo red binding of the amyloid suspension, employing stagnant solutions to form the fibrils in both cases (Figure 2A). Congo red binding also establishes the amyloid-like structure of the aggregates. The extent of L55P-TTR fibril formation observed at $48 \mathrm{~h}$ (Figure 2A) was generally greater than that observed at the $24 \mathrm{~h}$ time point, Figure 2B. Amyloid fibril formation (L55P-TTR) reached a plateau at $72 \mathrm{~h}$ for all suspensions evaluated over the $\mathrm{pH}$ range of $4.2-5.2$, making it difficult to identify the $\mathrm{pH}$ maximum at that time point (data not shown). Unlike wildtype TTR, which exhibits maximum amyloid fibril formation at $\mathrm{pH} 4.4$, L55P-TTR exhibits its maximum rate at $\mathrm{pH}$ 5.0. In addition, the extent of L55P-TTR amyloid fibril formation near physiological pHs (5.5-7.0) is much greater than that exhibited by wild-type TTR. As expected, good agreement was observed when comparing turbidity measurements and the Congo red binding as a measure of the extent of amyloid fibril formation, Figure 2, A and B.

The time course evaluation of the early stages of L55PTTR amyloid fibril formation as a function of $\mathrm{pH}$ revealed that amyloid fibril formation is significantly faster at $\mathrm{pH} 5.0$ than at $\mathrm{pH} 5.25$ or 4.4 , as detected by turbidity, Figure $2 \mathrm{C}$. It is important to realize that turbidity measurements do not detect small soluble assemblies that could artificially produce a lag phase even though self-assembly is occurring from the beginning, which is the case according to sedimentation velocity studies (16). The data shown in Figure 2C was acquired using a stirred assay, unlike the unstirred data shown in Figure 2A, B.

The L55P-TTR variant did not exhibit turbidity below $\mathrm{pH}$ $3.0(330 \mathrm{~nm})$ suggesting the absence of large insoluble aggregates, Figure 2, A and B. However, Congo red binding
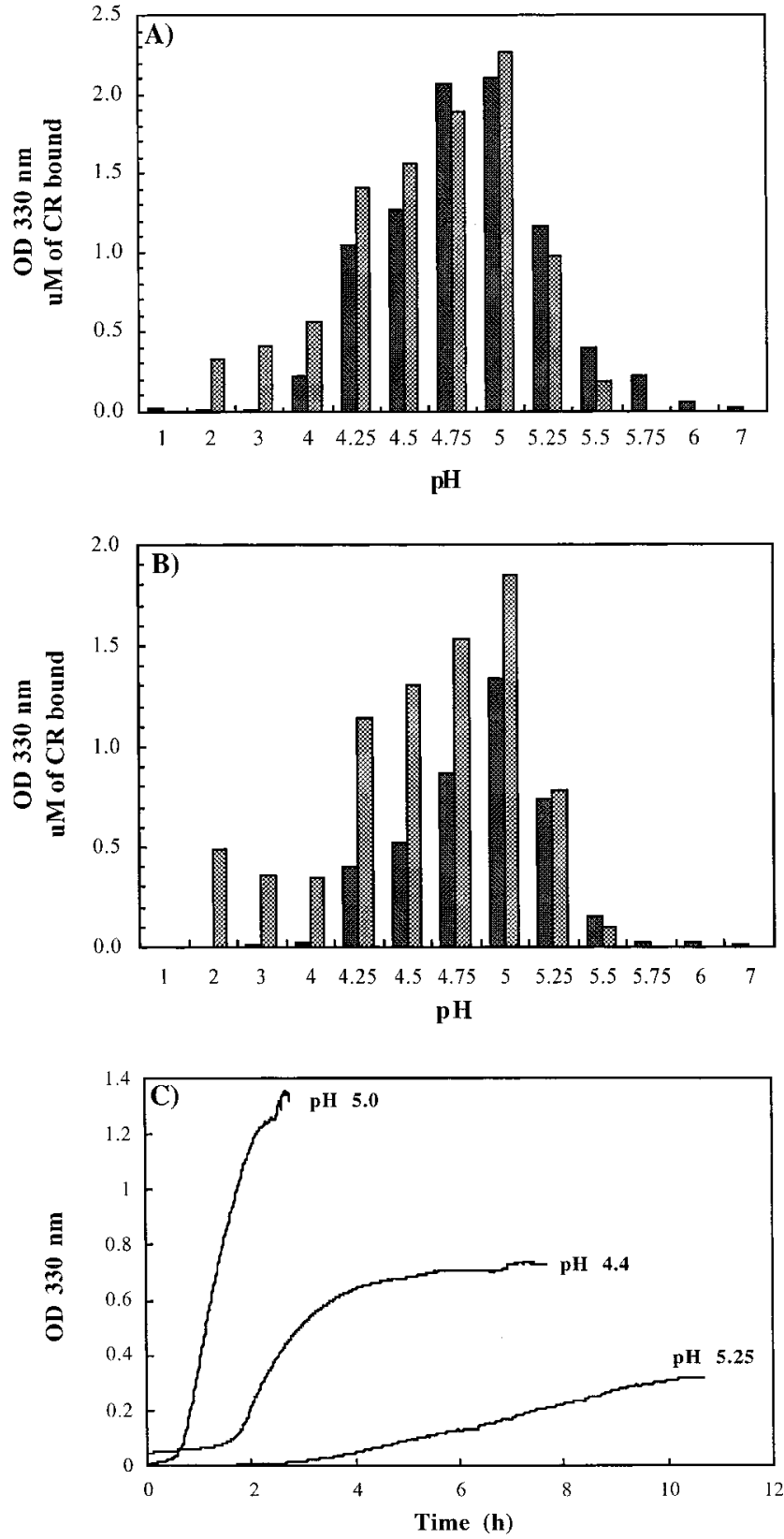

FIGURE 2: Bar graph depicting the extent of L55P-TTR fibril formation after $48 \mathrm{~h}$ (A) and $24 \mathrm{~h}(\mathrm{~B})$ as a function of $\mathrm{pH}$. The black bars represent the OD measurements while the light bars represent the extent of fibril formation based on a quantitative Congo red determination. (C) The time course of L55P-TTR selfassembly into protofilaments and filaments $(0.2 \mathrm{mg} / \mathrm{mL})$ as a function of $\mathrm{pH}(5.25,5.0$ and 4.4$)$ at $25^{\circ} \mathrm{C}$ monitored by turbidity $(330 \mathrm{~nm})$. This experiment demonstrates that L55P-TTR exhibits the maximal rate of fibril formation at $\mathrm{pH}$ 5.0.

was observed after incubation of TTR below pH 3.0 (Figure 2, A and B), suggesting the presence of smaller oligomers, at least some of which have an amyloid-like structure. The apparent discrepancy between the Congo red binding and the turbidity assays at lower pHs can be readily reconciled because the quaternary structures formed below $\mathrm{pH} 3$ are small and, therefore, not expected to exhibit turbidity as discerned from sedimentation velocity studies $(s=4-11 \mathrm{~S}$ (see below and Table 1; entry 11) (16).

Evaluating L55P-TTR Quaternary Structural Changes by Analytical Ultracentrifugation. Sedimentation equilibrium and velocity studies on L55P-TTR as a function of $\mathrm{pH}$, 
Table 1. Summary of Analytical Ultracentrifugation Experiments Carried Out with L55P-TTR as a Function of pH, Temperature, and Concentration $^{a}$

\begin{tabular}{|c|c|c|c|c|c|c|c|}
\hline entry & $\mathrm{pH}$ & $\begin{array}{l}\text { temp. } \\
\left({ }^{\circ} \mathrm{C}\right)\end{array}$ & $\begin{array}{l}\text { incubation } \\
\text { time }\end{array}$ & $\begin{array}{c}\text { concentration } \\
(\mathrm{mg} / \mathrm{mL})\end{array}$ & $\begin{array}{l}s \text { values and } M_{\mathrm{W}} \text { by } \\
\text { sedimentation velocity }\end{array}$ & $\begin{array}{c}M_{\mathrm{W}} \text { by } \\
\text { sedimentation } \\
\text { equilibrium }\end{array}$ & best fitting model \\
\hline 1 & 7 & 25 & 1-4 days & $0.2-1.0$ & $3.6-3.7 \mathrm{~S}(52-55 \mathrm{KDa})$ & $53 \mathrm{KDa}$ & tetramer \\
\hline 2 & 7 & 25 & $20-24 \mathrm{~h}$ & 0.02 & & $55 \mathrm{KDa}, 13 \mathrm{KDa}$ & tetramer, monomer \\
\hline 3 & $7^{b}$ & 37 & $>8$ weeks & 0.2 & $2-23 S$ & & $\sim 90 \%$ soluble oligomers \\
\hline 4 & $7^{c}$ & 37 & $2-8$ weeks & 0.2 & visible aggregates, $2-9 \mathrm{~S}, 3.7 \mathrm{~S}, 1.2 \mathrm{~S}$ & & soluble oligomers, tetramer, monomer \\
\hline 5 & $7^{d}$ & 4 & $\geq 2$ weeks & 0.2 & $9 \mathrm{~S}, 3.7 \mathrm{~S}(51 \mathrm{KDa}), 1.2 \mathrm{~S}(13.8 \mathrm{KDa})$ & & $20 \%$ oligomers and $80 \%$ (tetramer, monomer) \\
\hline 6 & $5.25-4.4^{e}$ & 25 & $20-24 \mathrm{~h}$ & 0.2 & extensive visible aggregation & & \\
\hline 7 & $5.25^{f}$ & 4 & $4 \mathrm{~h}$ & 0.2 & $3.7 \mathrm{~S}(54 \mathrm{KDa}), 1.4 \mathrm{~S}(14 \mathrm{KDa})$ & & tetramer, monomer \\
\hline 8 & 5 & 4 & $12 \mathrm{~h}$ & 0.2 & $2-9 \mathrm{~S}$ & & soluble oligomers \\
\hline 9 & 4.4 & 4 & $24 \mathrm{~h}$ & 0.2 & $4-13 \mathrm{~S}$ & & soluble oligomers \\
\hline 10 & 5 & 4 & $24 \mathrm{~h}$ & 0.02 & & $55,13.8 \mathrm{KDa}$ & tetramer, monomer \\
\hline 11 & $3.0-2.0^{g, h}$ & 4,25 & months & 0.2 & $4-11 \mathrm{~S}$ & $14-260 \mathrm{KDa}$ & A "state" like oligomers \\
\hline
\end{tabular}

${ }^{a}$ The L55P molecular weight under various conditions was determined by sedimentation equilibrium and/or sedimentation velocity $\left(M_{\mathrm{W}}\right.$ is calculated based on the diffusion coefficient values obtained by direct fitting of the data using Svedberg 5.0, which employs the following equation to calculate $M_{\mathrm{W}}=s R T / D(1-\bar{v} \rho)$. Samples were incubated at the indicated temperatures, but all sedimentation velocity experiments were carried out at $20{ }^{\circ} \mathrm{C}$. ${ }^{b}$ Reference 16. ${ }^{c}$ The amount of monomer left in solution increases with increasing incubation time and is highly dependent on salt (KCl) concentrations, with monomer making up $40-40 \%$ of the remaining soluble protein in samples that show extensive aggregation at $\mathrm{pH} 7\left(37^{\circ} \mathrm{C}\right) .{ }^{d}$ Samples incubated at $4{ }^{\circ} \mathrm{C}$ did not show any turbidity even after 4 weeks of incubation. ${ }^{e}$ Extensive aggregation and protein precipitation during the time scale of the analytical ultracentrifuge experiments precluded an accurate analysis of the data under these conditions. ${ }^{f}$ Sedimentation equilibrium data fits well only to a monomer-tetramer associating model for TTR; furthermore, the $M_{\mathrm{W}}$ vs concentration plot shows a $M_{\mathrm{W}}$ distribution of $17-46$ KDa. ${ }^{g}$ L55P forms soluble protofilaments that do not go on to form fibrils, even after incubation for months in vitro. ${ }^{h}$ L55P TTR is monomeric over the $\mathrm{pH}$ range $2-3$, in the absence of added $\mathrm{KCl}$.

temperature, and concentration reveal that L55P-TTR adopts a stable tetrameric structure over the physiological concentration range $\left(25^{\circ} \mathrm{C}, \mathrm{pH} 7\right)$ when incubated in solution for less than 4 days, Table 1 ; entry 1 . Sedimentation equilibrium results on L55P-TTR demonstrate that the tetramer starts to dissociate to monomer within $24 \mathrm{~h}$, yielding a tetramermonomer equilibrium at a concentration an order of magnitude below physiological $(0.02 \mathrm{mg} / \mathrm{mL}, \mathrm{pH} 7)$, Table 1 ; entry 2. Velocity studies on L55P-TTR at physiological concentrations $(0.2 \mathrm{mg} / \mathrm{mL})$ incubated at $\mathrm{pH} 7\left(37^{\circ} \mathrm{C}\right)$ for $>8$ weeks reveal that the protein does slowly dissociate to monomer, which then assembles into protofilaments under these conditions, the oligomers having $s$ values ranging from 2 to $23 \mathrm{~S}$, Table 1; entry 3. To demonstrate that L55P-TTR protofilament formation $(0.2 \mathrm{mg} / \mathrm{mL})$ likely occurs through the assembly of a monomeric amyloidogenic intermediate, sedimentation velocity experiments were carried out at $\mathrm{pH}$ 7 after incubation for 4 days at $37{ }^{\circ} \mathrm{C}$. The sedimentation velocity profiles reveal the presence of two resolvable boundaries in Figure $3 \mathrm{~A}$, each curve representing data as a function of time during sedimentation of the sample. Direct fitting of the sedimentation velocity profiles gives an excellent fit to a two species model with sedimentation coefficients and molecular weights that correspond to the L55P-TTR tetramer $\left(\mathrm{s}_{1}=3.7,53 \mathrm{KDa}, \sim 90 \%\right)$ and monomer $\left(\mathrm{s}_{2}=1.2,13 \mathrm{KDa}, \sim 10 \%\right)$. The appropriateness of the fit is demonstrated by the random residuals and the small standard deviations of \pm 0.015 AU shown in Figure 3B. Attempts to fit the data to a single ideal species model of tetramer, dimer, or monomer failed to give a good fit.

Incubation of L55P-TTR $(\mathrm{pH} 7)$ in the cold $\left(4{ }^{\circ} \mathrm{C}\right)$ at concentrations $\geq 0.2 \mathrm{mg} / \mathrm{mL}$ also leads to tetramer dissociation affording a monomer that assembles into protofilaments, Table 1; entry 5 . The sedimentation velocity profiles of a L55P-TTR sample $(0.2 \mathrm{mg} / \mathrm{mL})$ prepared from a $2 \mathrm{mg} / \mathrm{mL}$ stock solution that was stored at $4{ }^{\circ} \mathrm{C}$ for 3 weeks shows two resolvable boundaries; a fast sedimenting boundary that corresponds to protofilaments and a slow moving boundary that corresponds to a mixture of tetramer and monomer
(Figure 4A). The protofilaments formed after 1-4 weeks (4 ${ }^{\circ} \mathrm{C}$ ) constitute only $10-25 \%$ of the total protein content of the sample (absorbance analysis). The slow sedimenting boundary is composed predominantly of tetramer $(3.75 \mathrm{~S})$ and some monomer (1.4 S) 5-8\%, Figure 4B. Inclusion of the monomer in the fitting model was necessary to obtain a good fit for the data as determined by comparing the residuals from the fit to various models.

The L55P-FAP variant dissociates and self-assembles much faster over the $\mathrm{pH}$ range of maximal amyloid formation $\left(\mathrm{pH} 5.25-5.0 ; 25^{\circ} \mathrm{C}\right.$, Figure 2$)$ and within the time frame of the sedimentation velocity experiment $(2-4 \mathrm{~h})$, complicating data analysis and thus precluding an accurate evaluation of the quaternary structural species present in solution, Table 1; entry 6. Quaternary structural analysis by sedimentation velocity studies over this $\mathrm{pH}$ range were made possible by incubating the samples at $4{ }^{\circ} \mathrm{C}$ for $4 \mathrm{~h}$. Velocity analysis reveals that L55P-TTR ( $\mathrm{pH} \mathrm{5.25,} 4{ }^{\circ} \mathrm{C}$ ) exists as a mixture of tetramer $(s=3.7 \mathrm{~S}, 91 \%)$ and monomer (1.4 S, 9\%), Table 1; entry 7. Sedimentation velocity analysis of L55PTTR $(0.2 \mathrm{mg} / \mathrm{mL})$, incubated at $\mathrm{pH} 5.0\left(4{ }^{\circ} \mathrm{C}\right)$ for $12 \mathrm{~h}$, demonstrates the presence of a heterogeneous mixture of oligomeric species sedimenting with a distribution of sedimentation coefficients ranging from 2 to $9 \mathrm{~S}$, Table 1, entry 8. Sedimentation velocity studies of L55P-TTR under more acidic conditions $\left(\mathrm{pH} 4.4,4{ }^{\circ} \mathrm{C}\right)$ reduces the rate of aggregation, allowing the detection of a ladder of quaternary structure intermediates with $s$ values ranging from 4 to 13 $\mathrm{S}$; consistent with results reported previously for L55P-TTR (16), Table 1, entry 9.

To probe whether the monomeric amyloidogenic intermediate is in equilibrium with the functional tetramer or oligomers under acidic amyloid forming conditions, sedimentation equilibrium studies were carried out at lower TTR concentrations $(0.02 \mathrm{mg} / \mathrm{mL})$ at $4{ }^{\circ} \mathrm{C}$ to reduce the extent of self-assembly to protofilaments and fibrils. Attempts to fit sedimentation equilibrium data for L55P-TTR $(0.02 \mathrm{mg} / \mathrm{mL}$, $4{ }^{\circ} \mathrm{C}, \mathrm{pH} 5.0$ ) to a single ideal species model failed to give a good fit. However, an excellent fit was obtained to these 

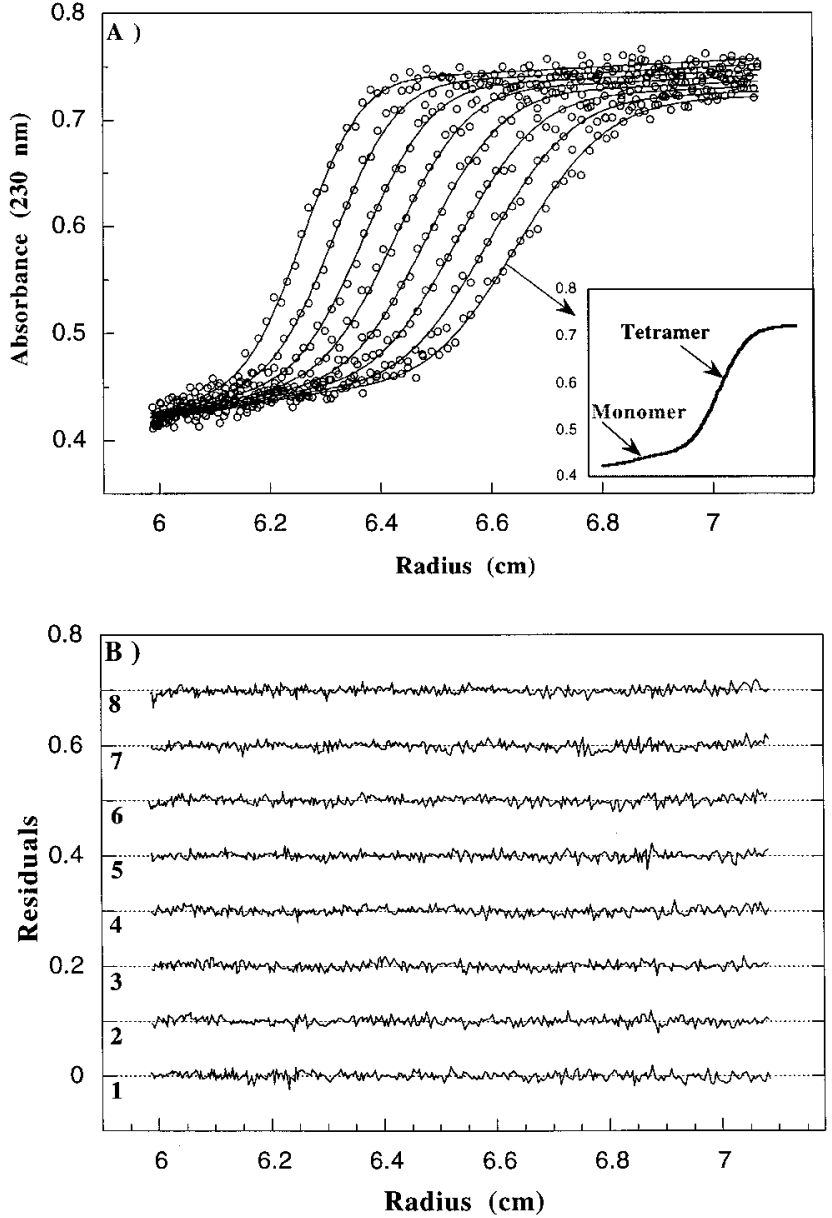

FIGURE 3: (A) Sedimentation velocity profiles of L55P-TTR $(0.2$ $\mathrm{mg} / \mathrm{mL}, \mathrm{pH}$ 7) incubated for 4 days at $37^{\circ} \mathrm{C}$. Scans for analysis were recorded every $3 \mathrm{~min}$; for clarity, only 8 representative scans 15 min apart are shown. The solid line represents the two-species fit (tetramer and monomer) to the experimental data $(\mathrm{O})$ using the Svedberg program. The inset in Figure A identifies the two sedimenting boundaries corresponding to the L55P-TTR tetramer and monomer. (B) The residuals (difference between the experimental data and the fitted data for each data point) for the two species fit. The overall root-mean-square deviation for the data is 0.015 absorbance units.

data using an associating monomer (13.8 KDa)-tetramer (55 $\mathrm{KDa}$ ) equilibrium model as judged by the residuals, Figure 5 , Table 1, entry 10 . The L55P-TTR variant, like the wildtype protein, assembles into small oligomeric structures over the $\mathrm{pH}$ range of 3.0-2.0, with sedimentation coefficients ranging from 4 to $11 \mathrm{~S}$, Table 1; entry 11 .

Evaluating L55P-TTR Quaternary Structural Changes by SDS-PAGE Analysis. Evaluation of the SDS-PAGE monitored L55P-TTR quaternary structure changes $(0.2 \mathrm{mg} / \mathrm{mL})$ as a function of $\mathrm{pH}$ is shown in Figure 6. The results demonstrate that L55P-TTR is predominantly tetrameric (runs as a dimer band) from $\mathrm{pH} 7.0$ to 5.5, Figure 6A. The L55P-TTR tetramer ( $\mathrm{pH}$ 7.0) is capable of undergoing slow dissociation to a monomer after $44 \mathrm{~h}$ of incubation at 37 ${ }^{\circ} \mathrm{C}$, as evident by the presence of a faint monomer band $(<10 \%)$ in the SDS-PAGE gel of the nonboiled TTR samples $(0.2 \mathrm{mg} / \mathrm{mL})$, Figure 6A. Below pH 5.0, the L55P-TTR monomer was the predominant species $(\sim 90-95 \%)$, with complete tetramer to monomer dissociation observed below $\mathrm{pH} 3.8$, Figure 6A. It is noteworthy that the monomeric band shown at pHs below 5.0 represents both L55P-TTR monomer
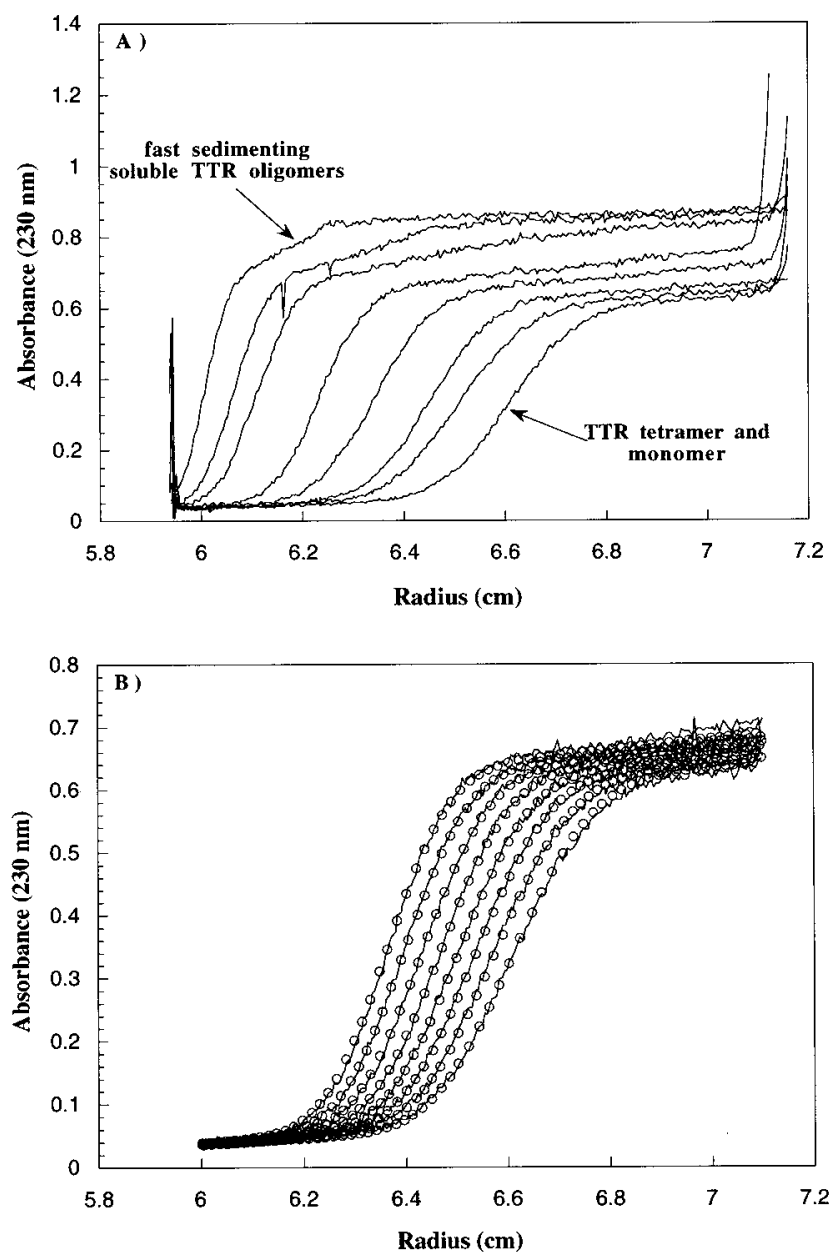

FIGURE 4: (A) Sedimentation velocity profiles of L55P-TTR $(0.2$ $\mathrm{mg} / \mathrm{mL}$ ) prepared by dilution from a stock solution of $2 \mathrm{mg} / \mathrm{mL}$ that was incubated at $4{ }^{\circ} \mathrm{C}$ for 4 weeks. The velocity data exhibits two resolvable boundaries, one corresponding to a mixture of soluble oligomeric species (amyloid precursors), with the second boundary corresponding to a mixture of predominantly L55P-TTR tetramer and 5-8\% monomer. (B) Direct fitting of the slow sedimenting boundary to a two species model of tetramer and monomer yields the two-species fit (solid line) to the experimental data $(\mathrm{O})$ using the Svedberg program.

and any SDS sensitive oligomeric species formed $(16,31)$. To evaluate whether the L55P-TTR tetramer-monomer transition observed over the $\mathrm{pH}$ range of 5-5.5 exhibits a concentration dependence, the quaternary structural changes were probed at three TTR concentrations, $0.2,0.1$, and 0.01 $\mathrm{mg} / \mathrm{mL}$, Figure 6B. At 0.2 and $0.1 \mathrm{mg} / \mathrm{mL}$, L55P-TTR exhibited similar acid dissociation transitions, with the L55P tetramer being the major species $(>80 \%)$ above $\mathrm{pH} 5.5$, below pH 5 the L55P monomer becomes the major species $(>90 \%)$. Interestingly, at a concentration of $0.01 \mathrm{mg} / \mathrm{mL}$, a significant fraction of the L55P-TTR tetramer had dissociated to monomer $(\approx 40 \%$, incubation time $=44 \mathrm{~h})$ above the $\mathrm{pH}$ range over which the acid mediated transition takes place (5.5-5) Figure 6, B and C, whereas L55P-TTR is predominantly tetrameric at physiological concentration $(0.2 \mathrm{mg} / \mathrm{mL})$ above pH 5.5. The results in Figure 6B demonstrate that the $\mathrm{pH}_{\mathrm{m}}(5.18)$ at all three concentrations $(0.2,0.1,0.01 \mathrm{mg} /$ $\mathrm{mL}$ ) was virtually identical, demonstrating that the tetramermonomer transition induced by acid denaturation is not very sensitive to TTR concentration, whereas the tetramermonomer equilibrium above $\mathrm{pH} 5.5$ is quite sensitive to 


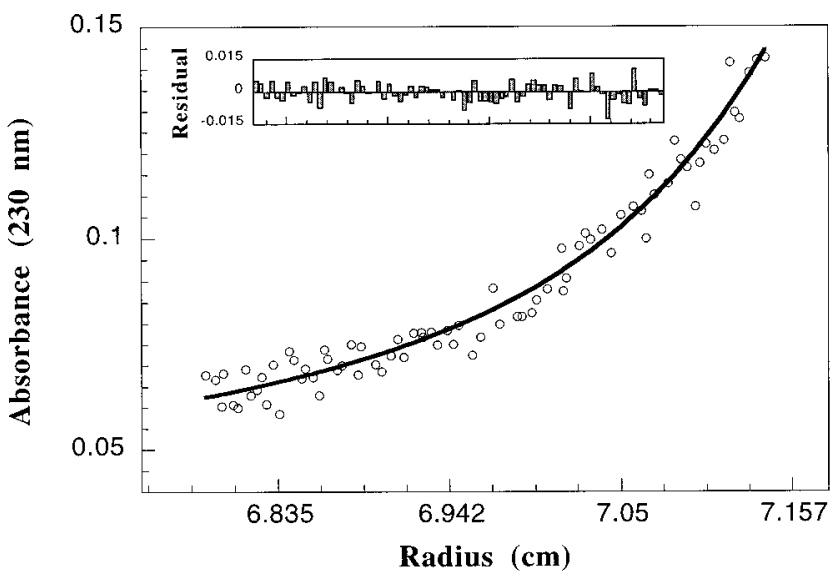

FIGURE 5: Sedimentation equilibrium analysis of L55P-TTR $(0.02$ $\mathrm{mg} / \mathrm{mL})$ in $50 \mathrm{mM}$ acetate buffer, $\mathrm{pH} 5.0\left(4^{\circ} \mathrm{C}\right)$. The solid line drawn through the data (o) was obtained by fitting the absorbance vs radial position data using a self-associating model for a monomer-tetramer equilibrium. The inset bar graph represent the residuals (difference between the experimental data and the fitted data for each point).

concentration. A time course evaluation of the L55P-TTR tetramer-monomer equilibrium at $\mathrm{pH} 7$ by SDS -PAGE analysis $(0.2$ and $0.01 \mathrm{mg} / \mathrm{mL})$ reveals no detectable changes in the amount of tetramer or monomer for incubation periods between 44 and $60 \mathrm{~h}$ (data not shown).

Monitoring Acid-Induced Denaturation of L55P-TTR by Fluorescence Spectroscopy. TTR amyloid fibril formation from the monomeric amyloidogenic intermediate is a concentration-dependent process that competes with denaturation at concentrations above $0.05 \mathrm{mg} / \mathrm{mL}$. Hence, $\mathrm{pH}$-dependent fluorescence experiments to monitor tertiary structural changes were carried out at a concentration of $0.01 \mathrm{mg} / \mathrm{mL}$, to prevent assembly of the amyloidogenic intermediate (fibril formation). Interestingly, L55P-TTR exhibits a biphasic acidmediated unfolding transition as monitored by tryptophan fluorescence (excitation at $295 \mathrm{~nm}$ ), Figure 7A. Over the $\mathrm{pH}$ range of $7.0-5.5$, the fluorescence intensities exhibited a slight fluctuation, but no significant change in the intensity, Figure 7A. From pH 5.5-5.0, the first transition was observed, which is likely the result of a linked quaternary and tertiary structural transition ( $\mathrm{pH}$-dependent quaternary structural changes are displayed in Figure 7A) to form the amyloidogenic intermediate. The fluorescence intensity of the tryptophans at pH 5.0 appeared to vary from one data set to another for reasons that remain unclear, but the overall shape of the acid-induced denaturation curve remains the same as indicated by the error bars for all data points (4 data sets) (see discussion). Over the $\mathrm{pH}$ range of 4.75-4.0, a slight increase in fluorescence intensity was observed followed by a plateau region from $\mathrm{pH} 4.0-3.5$. It is unclear whether the dip at $\mathrm{pH} 5$ is significant; what is clear is that the conformers populated over this $\mathrm{pH}$ range are amyloidogenic, Figure 2. Lowering the $\mathrm{pH}$ through the range of $3.5-2.5$ results in a second structural transition detected by fluorescence reflecting a transformation from the amyloidogenic intermediate(s) to a molten globule "A state", as discerned by ANS binding (see below), Figure 7A. This state is monomeric at low concentrations $(0.01 \mathrm{mg} / \mathrm{mL})$, but exists as an oligomer at higher concentrations $(0.2 \mathrm{mg} / \mathrm{mL})$ as discerned from its sedimentation properties evaluated previously $(16,31)$. At $\mathrm{pH} 3.0$, the emission maximum exhibited
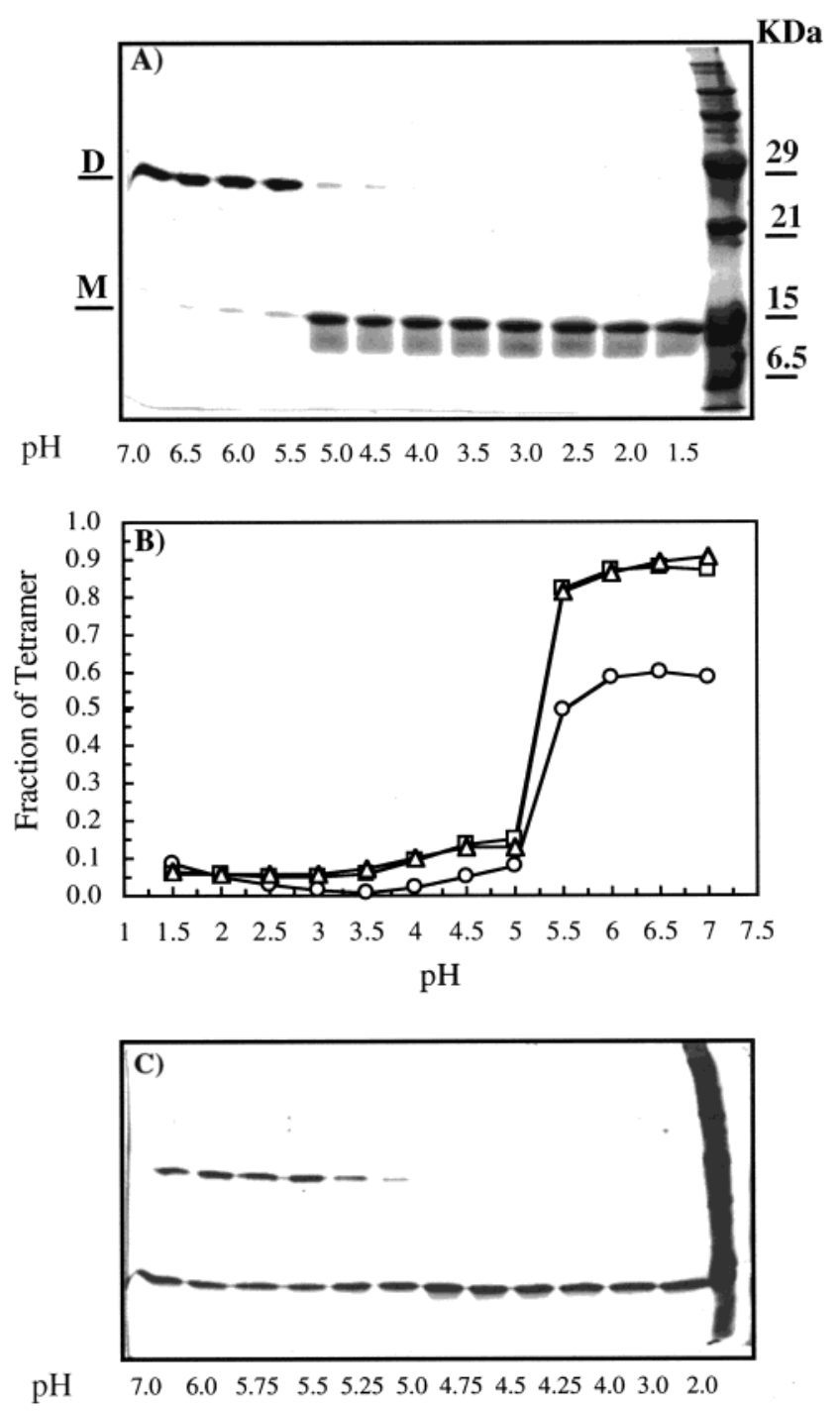

FIGURE 6: Concentration dependent quaternary structural changes in L55P-TTR as a function of $\mathrm{pH}$ (A) SDS-PAGE gel from which the L55P-TTR acid denaturation curve in Figure 6B was derived $(0.2 \mathrm{mg} / \mathrm{mL})$. (B) Tetramer-monomer transition as a function of $\mathrm{pH}$ for L55P-TTR at $0.2 \mathrm{mg} / \mathrm{mL}(\triangle), 0.1 \mathrm{mg} / \mathrm{mL}(\square)$ and 0.01 $\mathrm{mg} / \mathrm{mL}(\mathrm{O})$ after incubation for $40 \mathrm{~h}$ at $37^{\circ} \mathrm{C}$. (C) SDS-PAGE gel for L55P-TTR acid denaturation at $0.01 \mathrm{mg} / \mathrm{mL}$ employing smaller $\mathrm{pH}$ increments $(0.25 \mathrm{pH}$ unit steps).

a red shift from 337 to $342 \mathrm{~nm}$ indicating that the tryptophan residues in L55P-TTR are more, but not completely, exposed to solvent, Figure 7B. At pH 2, the fluorescence emission maximum showed a significant red shift to $355 \mathrm{~nm}$ and the fluorescence intensity decreased dramatically, most likely due to dynamic quenching as a result of further exposure of the side chains (unfolding) and increased side chain mobility, Figure 7B.

ANS Binding Monitoring Hydrophobic Surface Exposure. The fluorescence emission of ANS does not exhibit $\mathrm{pH}$ dependent behavior, therefore, any changes in the fluorescence spectra of ANS as a function of $\mathrm{pH}$ are a result of binding to exposed hydrophobic surfaces of L55P-TTR. It is known that two moles of ANS bind to the central thyroid binding channel of the WT-TTR tetramer, providing an internal stoichiometry calibration (63). Binding of ANS to the WT-tetramer is reported by an increase in ANS fluorescence as well as by a blue shift in the fluorescence emission maximum (31). ANS binding to L55P-TTR as a function of 

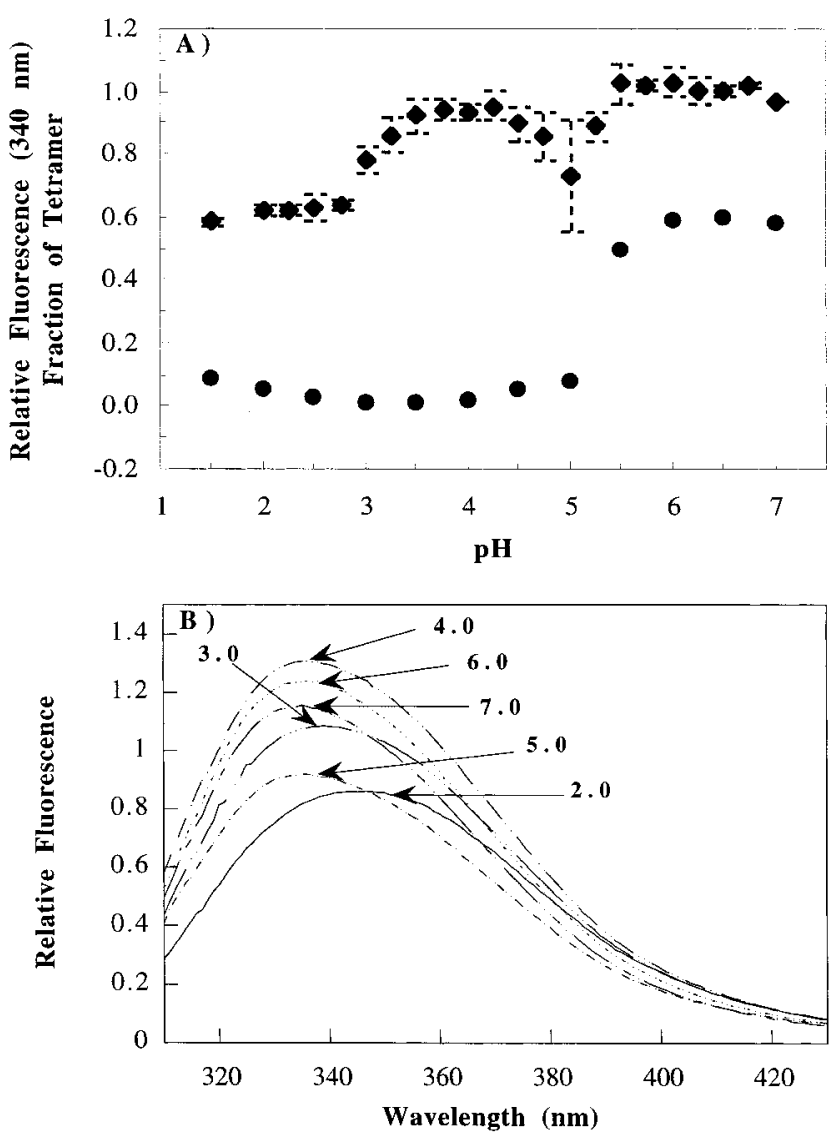

Figure 7: (A) Acid denaturation curve of L55P-TTR (0.01 mg/ $\mathrm{mL}$ ) monitored by changes in the tryptophan fluorescence intensity $(\diamond)$ at $340 \mathrm{~nm}$ (excitation at $295 \mathrm{~nm}$ ). The $\mathrm{pH}$ dependent equilibrium between tetramer and monomer as evaluated by the SDS-PAGE method is shown (O) to assist interpretation. (B) Tryptophan emission spectra of L55P-TTR $(0.01 \mathrm{mg} / \mathrm{mL})$ as a function of $\mathrm{pH}$ (excitation at $295 \mathrm{~nm}$ ).

$\mathrm{pH}$ is shown in Figure 8. Over the $\mathrm{pH}$ range of 7-6, ANS binding to the L55P-TTR tetramer is either very weak or it does not occur, Figure $8 \mathrm{~A}$. This can be rationalized because L55P-TTR exhibits a decreased affinity $(\approx 10 \times)$ for TTR ligands, and furthermore, only $60 \%$ of L55P-TTR is tetrameric at this concentration and $\mathrm{pH}$, Figure $6(23,64)$. The fluorescence emission spectrum of ANS in the presence of L55P-TTR at $\mathrm{pH} 5.0(520 \mathrm{~nm})\left(\mathrm{pH}_{\max }\right.$ for amyloid fibril formation) is equivalent to that exhibited by ANS alone in aqueous buffer, Figure $8 \mathrm{~B}$, consistent with the idea that the alternatively folded monomeric amyloidogenic intermediate formed does not bind to ANS. This behavior is similar to that of wild-type TTR, which exhibits a $\mathrm{pH}_{\max }$ for fibril formation and a minimum in ANS binding at $\mathrm{pH} 4.4$ (31). Below pH 5.0, ANS binding to L55P-TTR increases linearly with the decrease in $\mathrm{pH}$ (Figure 8), suggesting increased exposure of the hydrophobic surface as a likely result of a tertiary structural rearrangements in the L55P-TTR monomeric amyloidogenic intermediate, Figure 7, A and B. ANS fluorescence reached a maximum at $\mathrm{pH} 1.5$ where the monomer is largely unfolded.

Far and Near-UV CD of L55P-TTR as a Function of $p H$. The $\mathrm{pH}$-dependent conformational changes that allow L55PTTR amyloid fibril formation were probed by far- and nearUV CD studies at a concentration where assembly is not problematic $(0.01 \mathrm{mg} / \mathrm{mL})$. The $\mathrm{pH}$-dependent far-UV CD spectra of L55P-TTR shown in Figure 9A reveal that the
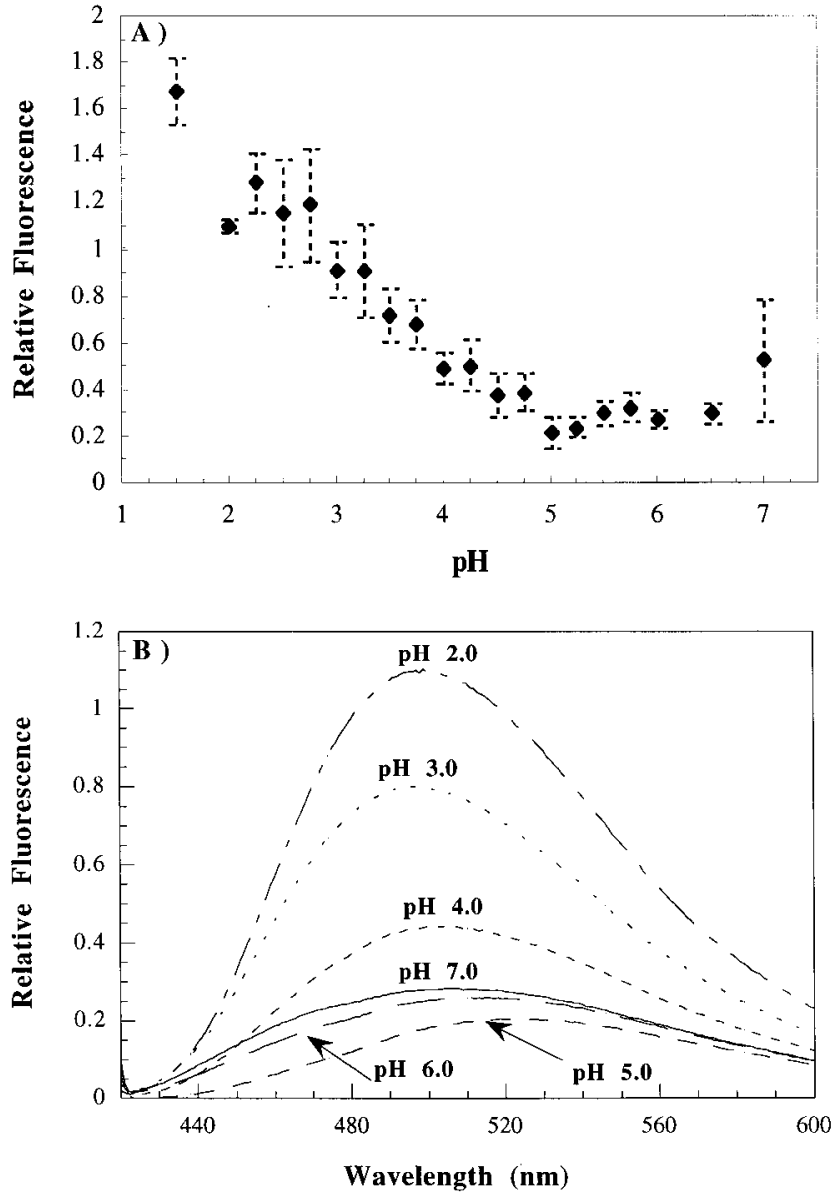

FIGURE 8: (A) Summary of ANS binding monitored by fluorescence emission at $490 \mathrm{~nm}$ as a function of $\mathrm{pH}$, error bars are shown to represent the standard deviation for 4 data sets. (B) ANS $(48 \mu \mathrm{M})$ binding to L55P-TTR $(0.01 \mathrm{mg} / \mathrm{mL})$ as a function of $\mathrm{pH}$ monitored by ANS fluorescence (excitation at $410 \mathrm{~nm}$ ) emission at $490 \mathrm{~nm}$.

secondary structural changes taking place under amyloid fibril formation conditions are subtle. At $\mathrm{pH} 7$ (native conditions), the far-UV CD of L55P-TTR exhibited a signal that is characteristic of $\beta$-sheet structure with a minimum at 214 and a maximum at $195 \mathrm{~nm}$. Upon lowering the $\mathrm{pH}$ to 5.0, the far-UV CD minimum for L55P-TTR does not change; however, a significant change in the maximum (195 $\mathrm{nm}$ ) intensity was observed (no detectable turbidity), Figure 9A. Although the amplitude of the two observable CD signals for $\beta$-sheet structure are dependent on sheet length, orientation, and twist, the absence of the maximum band at 195 $\mathrm{nm}$ in the far-UV CD signal may reflect a secondary structural rearrangement within the L55P-TTR monomer, leading to the formation of the monomeric amyloidogenic intermediate. This intermediate, which is significantly populated at $\mathrm{pH}$ 5.0, leads to maximal fibril formation at physiological concentration $(0.2 \mathrm{mg} / \mathrm{mL})$, Figure 2 . Over the $\mathrm{pH}$ range of $4-3$, L55P-TTR exhibits a broad and more intense minimum at $214 \mathrm{~nm}$, indicating further secondary structural changes. The L55P-TTR $(0.01 \mathrm{mg} / \mathrm{mL})$ minimum at $200 \mathrm{~nm}$ at $\mathrm{pH} 2$ in both the presence and absence of salt is consistent with a largely unordered ensemble of structures, Figure 9A.

The near-UV CD spectra of L55P-TTR, resulting from the asymmetric packing of the tryptophan and tyrosine side chains, revealed significant $\mathrm{pH}$-dependent tertiary structure changes, Figure 9B. The near-UV signals from the tryptophan 

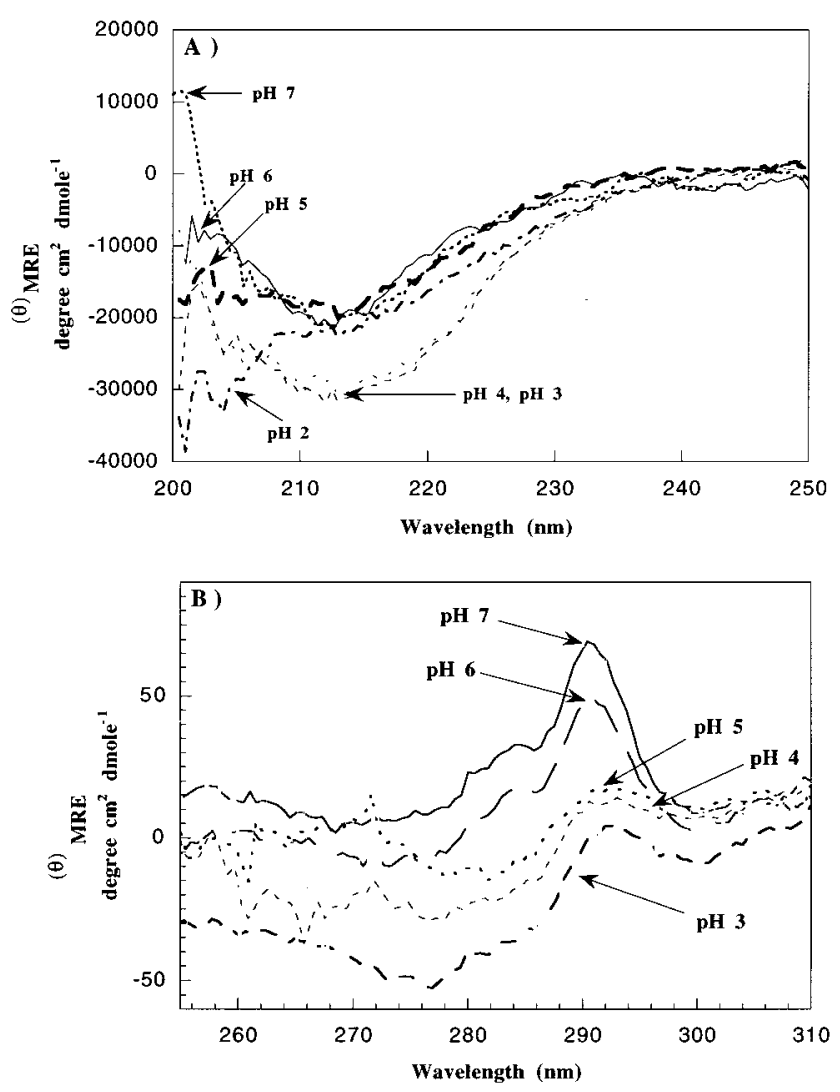

FIGURE 9: Conformational changes exhibited by L55P-TTR (0.01 $\mathrm{mg} / \mathrm{mL}$ ) monitored by far-UV CD (A) and Near-UV CD (B) as a function of $\mathrm{pH}$. Samples $(0.01 \mathrm{mg} / \mathrm{mL})$ were incubated for $2-6 \mathrm{~h}$ at $4{ }^{\circ} \mathrm{C}$ prior to collecting data to prohibit aggregation.

and tyrosine side chains at $\mathrm{pH} 7$ and 6 are not significantly different, in agreement with the far-UV data and consistent with the absence of significant Trp fluorescence changes over this $\mathrm{pH}$ range. Near-UV CD change was observed at $\mathrm{pH} 5.0$ where a significant decrease in intensity of the near-UV CD signal for both tryptophan $(292 \mathrm{~nm})$ and tyrosine $(284 \mathrm{~nm})$ residues was detected, consistent with the formation of the amyloidogenic intermediate, Figure 9B. At $\mathrm{pH} 4.0$, the nearUV CD signal for both tryptophan and tyrosine residues decreased further, consistent with the further tertiary structural rearrangement. Over the $\mathrm{pH}$ range of 4.0-3.0, the tryptophan near-UV CD is diminished further and the spectra exhibits a broad minimum around $275 \mathrm{~nm}$, suggesting that L55P-TTR adopts a less ordered tertiary structure at this $\mathrm{pH}$. At $\mathrm{pH}$ 2.0, a dramatic change was observed in the near-UV CD spectra, consistent with a more unfolded structure (data not shown). These results suggest a tertiary structural change in L55P-TTR occurring over the $\mathrm{pH}$ range of $6-5$, with an additional modest tertiary structural change between $\mathrm{pH} 5-4$, followed by a significant tertiary structural change when the $\mathrm{pH}$ is lowered further toward $\mathrm{pH}$ 2.0, Figure 9B.

Electron Microscopy to Follow the Assembly of the Amyloidogenic Intermediate. Blake and co-workers have previously shown that TTR amyloid fibrils isolated from the vitreous humor of the eye are uniform, straight, and unbranched fibers of approximately $100-130 \AA$ in diameter by electron microscopy (39). A single transthyretin amyloid fibril is made up of four filaments, each having a characteristic cross- $\beta$ repeat structure that is about $50-60 \AA$ in diameter. The observation of smaller oligomeric intermedi- ates during the process of transthyretin amyloid formation in vitro was not reported until recently (16). The presence of oligomeric, putative amyloid fibril precursors has also been detected in $\mathrm{A} \beta$ fibrillogenesis by several groups (15, $17,18,20,65)$. The dependence of L55P-TTR protofilament formation on solution conditions, such as ionic strength, $\mathrm{pH}$, and temperature, was evaluated here. Briefly, these studies reveal the formation of L55P-TTR protofilaments after 1 week of incubation at $37^{\circ} \mathrm{C}(\mathrm{pH} 6.0-7.5,0-200 \mathrm{mM} \mathrm{KCl})$. Protofilament formation was less efficient at $\mathrm{pH} 6.0$ than over the $\mathrm{pH}$ range of $6.5-7.5$. An electron microscopy time course study at $\mathrm{pH} 7.5\left(37^{\circ} \mathrm{C}\right)$ demonstrates that L55P-TTR is initially tetrameric, exhibiting an average diameter of 50$55 \AA$ (Figure 10A). After 1 week of incubation, a slow conversion of the tetramer into monomer ( $37 \AA$ diameter) and finally into protofilaments having an average diameter of $60-65 \AA$, with an average length of $50 \mathrm{~nm}$ (Figure 10B) is observed. These short protofilaments elongate slowly (11.5 months) generating protofilaments with an average length of $110 \mathrm{~nm}$, Figure 10C. The L55P-TTR protofilaments bind thioflavin $\mathrm{T}$ and Congo red, albeit more weakly than do fibrils formed under acidic conditions.

\section{DISCUSSION}

Wild-type transthyretin amyloid fibril formation appears to require tetramer dissociation to an alternatively folded monomer (amyloidogenic intermediate), which then selfassembles into numerous quaternary structural intermediates (protofilaments and filaments), ultimately affording amyloid fibrils $(1,22,31,33)$. Studies described in the previous section demonstrate that the familial amyloid polyneuropathy-associated variant L55P also requires tetramer disassembly and subtle conformational changes within the monomeric subunit to be competent to undergo self-assembly $(16,29,30,52,66)$. Several biophysical methods, including analytical ultracentrifugation studies, are able to detect L55P self-assembly when the monomer is also detected in solution, but self-assembly (amyloid formation) is not observed when the tetramer is exclusively populated. The dimeric form of transthyretin has not been detected in significant amounts $(<3 \%)$ in the case of WT-TTR or the FAP-associated variants studied thus far under conditions where amyloid fibril formation is also observed, suggesting that either the tetramer directly dissociates to monomer or that the dimer does not build up to a detectable concentration (16). Dissociation of the WT-TTR tetramer to monomer is not observed under physiological conditions $(0.2 \mathrm{mg} / \mathrm{mL}, \mathrm{pH} 7)$. In contrast, L55P-TTR slowly dissociates from its tetrameric structure, yielding a monomer under physiological conditions (pH 7, $37^{\circ} \mathrm{C} .0 .2 \mathrm{mg} / \mathrm{mL}$ ) that subsequently self-assembles into protofilaments, Figure 11. The activation barrier for L55P-TTR dissociation is accessible under physiological conditions in vitro (Figure 3), whereas the dissociation barrier for WT and V30M TTR are not surmountable on a biologically relevant time scale at $37^{\circ} \mathrm{C}([\mathrm{TTR}]=0.2 \mathrm{mg} /$ $\mathrm{mL)}$ (66).

It appears that two different mechanisms of L55P-TTR tetramer dissociation are operational for affording the L55PTTR monomeric amyloidogenic intermediate, i.e. there are concentration and $\mathrm{pH}$-dependent pathways. The tetramermonomer equilibrium is dependent upon concentration above $\mathrm{pH}$ 5.5; a concentration an order of magnitude below 

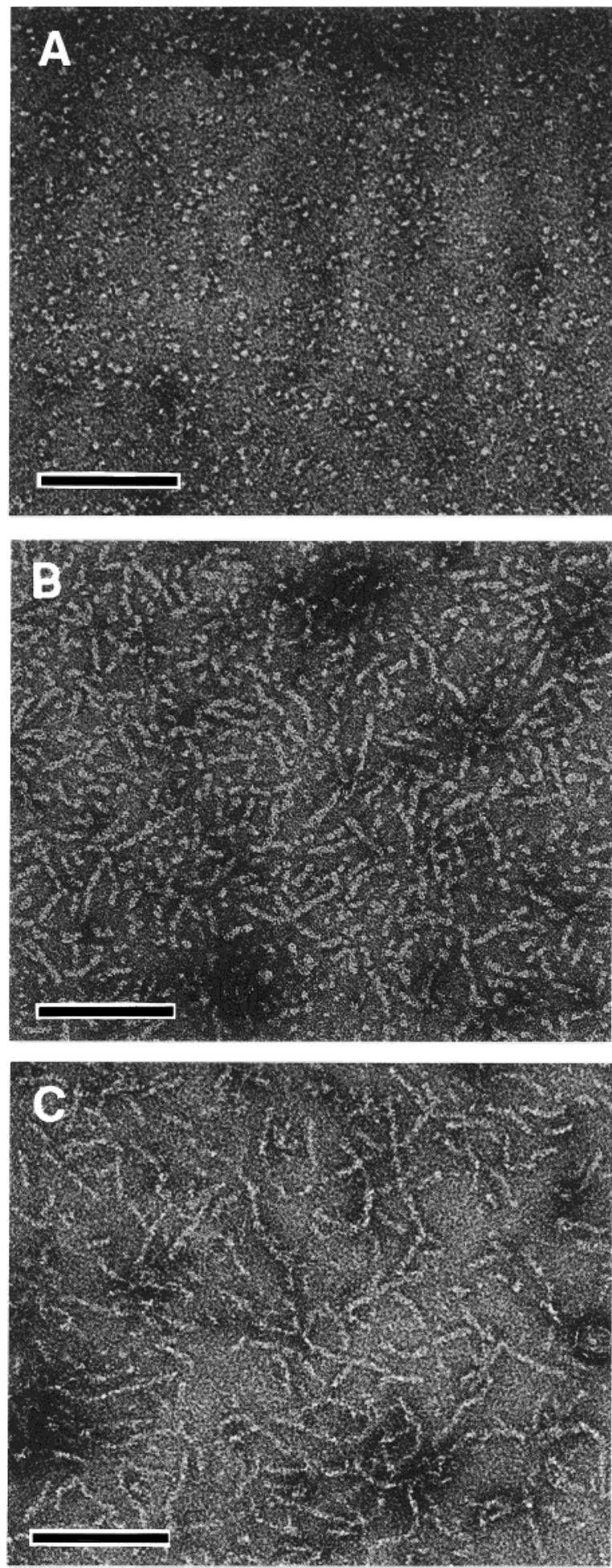

FIGURE 10: (A) Electron micrograph of L55P-TTR after $24 \mathrm{~h}$ of incubation under simulated physiological conditions $(0.2 \mathrm{mg} / \mathrm{mL}$, $100 \mathrm{mM} \mathrm{KCl}, \mathrm{pH} 7.5,37^{\circ} \mathrm{C}$ ) in vitro representing predominantly L55P-TTR tetramer (having a diameter of $50-55 \AA$ ) and $<3 \%$ monomer (see Table 1), exhibiting a diameter of $37 \AA$ (B) Electron micrograph of L55P-TTR $(0.2 \mathrm{mg} / \mathrm{mL}, 100 \mathrm{mM} \mathrm{KCl}, \mathrm{pH} 7.5)$ incubated at $37{ }^{\circ} \mathrm{C}$ for 1 week revealing abundant quantities of short assembled L55P-TTR protofilaments having an average diameter of $60-65 \AA$ and an average length of $50 \mathrm{~nm}$ (C) Electron micrograph of elongated L55P-TTR protofilaments $(110 \mathrm{~nm}$ average length) that form at $37^{\circ} \mathrm{C}$ after $1-1.5$ months of incubation. For all micrographs, the scale bar $=100 \mathrm{~nm}$, magnification is $145000 \times$.

physiological $(0.01 \mathrm{mg} / \mathrm{mL})$ affords $40 \%$ L55P-TTR monomer, whereas $\approx 15 \%$ monomer is observed for wild-type TTR under these conditions (31). At physiological concentration $(0.2 \mathrm{mg} / \mathrm{mL}), \mathrm{L} 55 \mathrm{P}-\mathrm{TTR}$ is greater than $97 \%$ tetrameric $(\mathrm{pH}$ $7,25{ }^{\circ} \mathrm{C},<96 \mathrm{~h}$ ). The effect of $\mathrm{pH}$ on the L55P-TTR tetramer-monomer equilibrium is dramatic over the range of 5.5 to 5 . A pH of 5 nearly completely dissociates the L55P tetramer, a pH that does not dissociate the WT-TTR tetramer. The protonation of one or more carboxylate or imidazole side chains with an apparent $\mathrm{p} K_{\mathrm{a}}$ of 5.18 mediates a likely conformational change that efficiently orchestrates L55PTTR tetramer dissociation, Figure 6. The $\mathrm{pH}$-mediated tetramer dissociation is largely independent of TTR concentration, except for the fact that the TTR concentration sets the pretransition tetramer/monomer ratio. The fact that maximal amyloid fibril formation is observed at $\mathrm{pH} 5$, where the monomer is maximally populated and structured, is consistent with the importance of the monomeric amyloidogenic intermediate in amyloid fibril formation.

Evidence for a defined, but alternatively folded L55P-TTR monomeric amyloidogenic intermediate is quite strong. Two $\mathrm{pH}$-mediated tertiary structural changes monitored by fluorescence are detected at a concentration where self-assembly is minimal, Figure 7. The first linked quaternary and tertiary structural transition, $\mathrm{pH}_{\mathrm{m}}$ 5.18, yields the amyloidogenic intermediate, while the second tertiary structural transition centered around $\mathrm{pH} 3.25$, clearly destroys the structure required for self-assembly and ultimately amyloid fibril formation, Figures 2 and 7 . It is interesting that the fluorescence data point at $\mathrm{pH} 5$, the $\mathrm{pH}$ of maximal amyloid fibril formation, exhibits variability. This experiment has been repeated several times with the standard deviation shown in Figure 7. Possibilities for the considerably larger standard deviation than for other data points include the fact that the tetramer-monomer equilibrium and the tertiary structural change populating the amyloidogenic intermediate are linked and appear to be hypersensitive to conditions at this $\mathrm{pH}$. There also may be variable amounts of assembly occurring at $\mathrm{pH} 5$ that we simply cannot detect, likely due to the isoelectric point for the L55P-TTR monomer being very close to this $\mathrm{pH}$. Undoubtedly, this variability is telling us something about the monomeric amyloidogenic intermediate, though we cannot fully appreciate its meaning at this time. The $\mathrm{pH}$ range over which the acid dissociation transition $\left(\mathrm{pH}_{\mathrm{m}}=5.18\right)$ observed for L55P-TTR takes place is much narrower than that reported previously, likely due to the presence of undetected soluble L55P-TTR oligomers in the protein stock solutions used for earlier studies (30).

There may well be an additional tertiary structural transition occurring over the $\mathrm{pH}$ range of 5-4.25, i.e. the amyloidogenic intermediate may be in equilibrium with a more disordered structure. This hypothesis is supported by considering both the far- and near-UV CD spectra that exhibit modest changes when the $\mathrm{pH}$ is dropped below 5 and more dramatic changes at $\mathrm{pH} 4$, Figure 9. The increased ANS binding (fluorescence) over the $\mathrm{pH}$ range of 5 (maximal fibril formation) to 4 (reduced fibril formation) also supports a conformation change associated with increased hydrophobic exposure. The fluorescence transition associated with destruction of the amyloidogenic intermediate centered around $\mathrm{pH} 3.25$ exposes significant hydrophobic surface area as discerned from ANS binding, Figure 8 (67).

From a comparison of the tetramer stabilities of WT-TTR to the FAP-associated variants V30M and L55P, it is clear 
that L55P-TTR is most sensitive to acidic dissociation, followed by the V30M variant, followed by the WT protein, as discerned from previous studies $(16,30,31,33)$. The effect of concentration on the tetramer-monomer equilibrium at neutral $\mathrm{pH}$ is not easily detected for WT-TTR or for the FAP variant V30M, whereas the effect is significant and easily detected for L55P-TTR. Transthyretin is a negative acute phase reactant protein, meaning that its concentration is reduced during inflammation and/or malnourishment (68). A drop in L55P-TTR concentration in response to these stimuli could actually increase the concentration of the amyloidogenic intermediate at neutral $\mathrm{pH}$ and could play a role in familial amyloid polyneuropathy under inflammatory stress, especially when the L55P variant is involved.

Wild-type and V30M TTR will only form fibrils efficiently under acidic conditions, suggesting late endosome or lysosome involvement in TTR amyloid diseases (29). It appears that the activation barrier for WT and V30M-TTR tetramer dissociation is simply not surmountable under physiological conditions $(16,31)$. On the other hand, the L55P FAPassociated variant will dissociate and form the monomeric amyloidogenic intermediate that assembles into protofilaments under physiological conditions, suggesting that L55PTTR fibrils could form in the extracellular space as well as in intracellular (e.g., lysosomal) locations (pH-mediated) (16). Importantly, the extent of L55P-TTR amyloid fibril formation is dramatically enhanced under acidic conditions, Figure 2, leading one to speculate that an acidic environment may be the physiologically relevant means of amyloid formation in vivo, especially when the half-life of transthyretin is considered (maximally $72 \mathrm{~h}$ ). Only traces of monomer are produced over this time frame as can be seen in Figures 3 and 6 at $\mathrm{pH}$ 7.0. However, the half-life of L55P-TTR in plasma becomes irrelevant if the protofilaments resulting from its assembly can avoid turnover. Cell biological studies currently underway in the laboratory will address these possible scenarios directly.

It is not yet clear how closely the protofilaments formed at $\mathrm{pH} 7.0$ are in structure to the filaments composing the amyloid fibrils; however, they do bind Congo red and thioflavin T, albeit more weakly. Further studies are necessary to compare their structures and to decide which of these structures are relevant to pathology.

A common mechanism has emerged for the conversion of wild-type, V30M and L55P transthyretin into amyloid fibrils; however, the kinetics and thermodynamics of this process differ significantly for L55P-TTR relative to WT and V30M-TTR. In all cases, the functional tetrameric form of transthyretin dissociates under acidic conditions into a monomeric amyloidogenic intermediate that self-associates into protofilaments, filaments, and fibrils, Figure 11. The activation barrier for L55P-TTR tetramer dissociation is surmountable under physiological conditions, which is not the case for WT- or V30M-TTR. That this is the case is easily discernible from time dependent TEM studies (Figure 10) and the observation that WT- and V30M-TTR exhibit hysteresis when subjected to $\mathrm{Gdn}-\mathrm{HCl}$-induced unfolding and dilution induced refolding, unlike L55P where the folded and unfolded states are in equilibrium in the transition region (66). The L55P-TTR tetramer is less stable than the WTTTR tetramer under physiological and acidic conditions as well as toward denaturants, such as Gdn-HCl. The L55P-
L55P-TTR Forms Protofilaments Under Physiological Conditions and Amyloid Under Acidic Conditions.

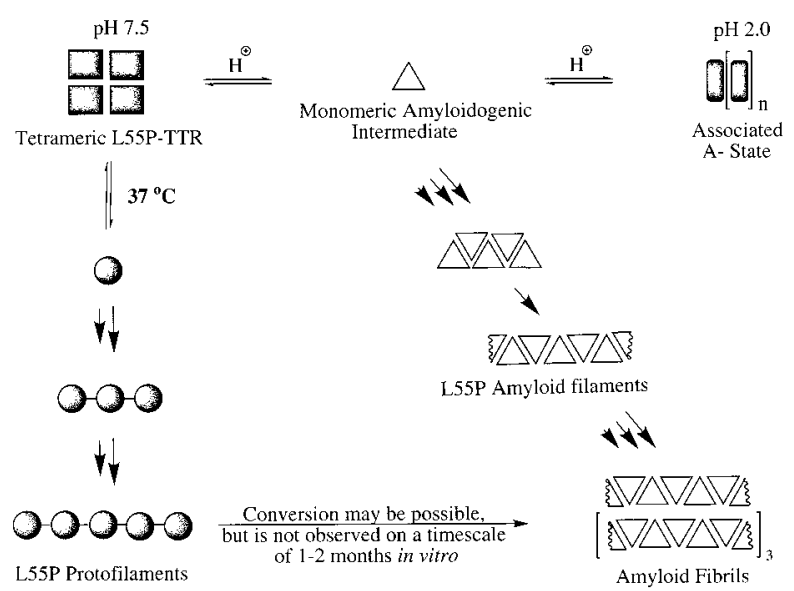

Structural Similarity between $\bigcirc$ and $\triangle$ is likely, but unclear at this time.

FIGURE 11: Summary of our current understanding of the amyloidogenic properties of L55P-TTR.

TTR tetramer is predisposed to form the amyloidogenic intermediate owing to favorable kinetics and thermodynamics. Since tetramer dissociation appears to be the first committed step in amyloid fibril formation, the small molecule approach developed earlier by this laboratory to prevent amyloid fibril formation should be quite effective for reducing or eliminating amyloid fibril formation in familial amyloid polyneuropathy associated with L55P-TTR $(23-26)$. One advantage to the slow in vitro conversion of protofilaments into fibrils exhibited by L55P-TTR at neutral $\mathrm{pH}$ (Figure 11) is that it may be possible to eliminate the fibrils as the key suspect in the neurodegeneration using this variant. If this holds true in cell culture experiments, then it may be possible to narrow in on the culprit of neurodegeneration, i.e. to shed light on the key structural entity or entities in neurodegeneration.

\section{CONCLUSION}

The L55P FAP-associated TTR variant tetramer-monomer equilibrium exhibits greater sensitivity to acidification and protein concentration (above $\mathrm{pH} 5.5$ especially) than either the WT or V30M FAP variant. The ability of L55PTTR to dissociate to an amyloidogenic monomer under conditions where WT and V30M TTR exhibit a stable tetrameric structure seems to explain the extreme pathogenicity of the L55P variant. Subtle tertiary structural changes in the L55P monomer appear to enable self-assembly of this intermediate into protofilaments. Importantly, these results suggest a common mechanism for TTR amyloid fibril formation and, therefore, a common therapeutic strategy can be envisioned for preventing fibril formation in the diseases SSA and FAP, even though the kinetic barriers for L55P and WT-TTR differ dramatically $(1,16,66)$.

\section{ACKNOWLEDGMENT}

We are grateful to Dr. Edward Koepf and Dr. Prakash Raman for helpful discussions and Dr. Roberto De Guzman and Dr. Gayathri Ratnaswamy for preparing the Molscript figure. 


\section{REFERENCES}

1. Kelly, J. W. (1996) Curr. Op. Struct. Biol. 6, 11-17.

2. Jacobson, D. R., and Buxbaum, J. N. (1991) Adv. Human Genetics 20, 69-123.

3. Sipe, J. D. (1992) Annu. Rev. Biochem. 61, 947-975.

4. Cornwell, G. C., Sletten, K., Johansson, B., and Westermark, P. (1988) Biochem. Biophys. Res. Comm. 154, 648-653.

5. Gustavsson, A., Engstrom, U., and Westermark, P. (1991) Biochem. Biophys. Res. Comm. 175, 1159-1164.

6. Kelly, J. W., and Lansbury, P. T. J. (1994) Amyloid: Int. J. Exp. Clin. Invest. 1, 186-205.

7. Selkoe, D. J. (1997) Science 275, 630-631.

8. Jacobson, D. R., Pastore, R. D., Yaghoubian, R., Kane, I., Gallo, G., Buck, F. S., and Buxbaum, J. N. (1997) N. Engl. J. Med. 336, 466-73.

9. Howlett, D. R., Jennings, K. H., Lee, D. C., Clark, M. S. G., Brown, F., Wetzel, R., Wood, S. J., Camilleri, P., and Roberts, G. W. (1995) Neurodegeneration 4, 23-32.

10. Pike, J. C., Walencewicz-Wasserman, J. C., Kosmoski, H. D., Cribbs, H. D., Glabe, G. C., and Cotman, W. C. (1995) J. Neurochem. 64, 253-265.

11. Burdick, D., Kosmoski, J., Knauer, M. F., and Glabe, C. G. (1997) Brain Res. 746, 275-284.

12. Hardy, J., and Allsop, D. (1991) TIPS 12.

13. Hardy, J. A., and Higgins, G. (1992) Science 256, 184-185.

14. Lansbury, P. T. (1999) Proc. Natl. Acad. Sci. U.S.A. 96, 33423344.

15. Harper, J. D., Wong, S. S., Lieber, C. M., and Lansbury, P. T., Jr. (1997) Chem. Biol. 4, 119-125.

16. Lashuel, H. A., Lai, Z., and Kelly, J. W. (1998) Biochemistry $37,17851-17864$.

17. Walsh, D. M., Lomakin, A., Benedek, G. B., Condron, M. M., and Teplow, D. B. (1997) J. Biol. Chem. 272, 2236422372.

18. Lambert, M. P., Barlow, A. K., Chromy, B. A., Edwards, C., Freed, R., Liosatos, M., Morgon, T. E., Rozovsky, I., Trommer, B., Viola, K. L., Wals, P., Zhang, C., Finch, C. E., and Krafft, G. A. (1998) Proc. Natl. Acad. Sci. U.S.A. 95, 6448-6453.

19. Giordano, T. J., Pan, L. M., Monteggia, L. M., Holzman, S. W., Snyder, G. A., Kraft, G. A., Ghanbari, H., and Kowall, N. W. (1994) Exp. Neurol. 125, 175-182.

20. Snyder, S. W., Ladror, U. S., Wade, W. S., Wang, G. T., Barrett, L. W., Matayoshi, E. D., Huffaker, H. J., Krafft, G. A., and Holzman, T. F. (1994) Biophys. J. 67, 1216-1228.

21. Kelly, J. W. (1998) Curr. Op. Struct. Biol. 8, 101-106.

22. Kelly, J. W. (1997) Structure 5, 595-600.

23. Peterson, S. A., Klabunde, T., Lashuel, H. A., Purkey, H., Sacchettini, J. C., and Kelly, J. W. (1998) Proc. Natl. Acad. Sci. U.S.A. 95, 13407-13412.

24. Miroy, G. J., Lai, Z., Lashuel, H., Peterson, S. A., Strang, C., and Kelly, J. W. (1996) Proc. Natl. Acad. Sci. U.S.A. 93, 15051-15056.

25. Baures, P. W., Peterson, S. A., and Kelly, J. W. (1998) Bioorg. Med. Chem. 6, 1389-1401.

26. Baures, P. W., Oza, V. H., Peterson, S. A., and Kelly, J. W. (1999) Biorg. Med. Chem. Lett. 9, 1-6.

27. Lansbury, P. T. (1992) Biochemistry 31, 6865-6870.

28. Sunde, M., Serpell, C. S., Bartlam, M., Fraser, E. P., Pepys, B. M., and Blake, C. F. C. (1997) J. Mol. Biol. 273, 729739.

29. McCutchen, S., Colon, W., and Kelly, J. W. (1993) Biochemistry 32, 12119-12127.

30. McCutchen, S. L., Lai, Z., Miroy, G., Kelly, J. W., and Colon, W. (1995) Biochemistry 34, 13527-13536.

31. Lai, Z., Colon, W., and Kelly, J. W. (1996) Biochemistry 35, 6470-6482.

32. Glenner, G. G., Ein, D., Eanes, E. D., Bladen, H. A., Terry, W., and Page, D. L. (1971) Science 174, 712-714.

33. Colon, W., and Kelly, J. W. (1992) Biochemistry 31, 86548660 .
34. Booth, D. R., Sunde, M., Bellotti, V., Robinson, C. V., Hutchinson, W. L., Fraser, P. E., Hawkins, P. N., Dobson, C. M., Radford, S. E., Blake, C. C. F., and Pepys, M. B. (1997) Nature 385, 787-793.

35. Wetzel, R. (1991) in Stability of Protein Pharmaceuticals: In Vivo Pathways of Degradation and Strategies for Protein Stabilization (Ahern, T., and Manning, M., Eds.), Plenum, NY, NY.

36. Helms, L. R., and Wetzel, R. (1996) J. Mol. Biol. 257, 7786.

37. Hurle, M. R., Helms, L. R., Li, L., Chan, W., and Wetzel, R. (1994) Proc. Natl. Acad. Sci. U.S.A. 91, 5446-5450.

38. Blake, C. C. F., Geisow, M. J., and Oatley, S. J. (1978) J. Mol. Biol. 121, 339-356.

39. Blake, C., and Serpell, L. (1996) Structure 4, 989-998.

40. Saraiva, M. J. M. (1995) Hum. Mutat. 5, 191-196.

41. Westermark, P., Sletten, K., Johansson, B., and Cornwell, G. G. (1990) Proc. Natl. Acad. Sci. U.S.A. 87, 2843-2845.

42. Saraiva, M. J. M., Costa, P. P., and Goodman, D. S. (1983) J. Lab. Clin. Med. 102, 590-603.

43. Saraiva, M. J. M., Birken, S., Costa, P., and Goodman, D. S. (1984) J. Clin. Inv. 74, 104-119.

44. Saraiva, M. J., Costa, P. P., and Goodman, D. S. (1985) J. Clin. Invest. 76, 2171-77.

45. Saraiva, M. J. M., Costa, P. P., and Goodman, D. S. (1988) Adv. Neurol. 48, 189-200.

46. Benson, M. D., and Wallace, M. R. (1989) in The Metabolic Basis of Inherited Disease (Scriver, C. R., Beaudet, A. L., Sly, W. S., and Valle, D., Eds.) pp 2439, McGraw-Hill, NY, NY.

47. Schormann, N., Murrel, R. J., and Benson, D. M. (1998) Amyloid: Int. J. Exp. Clin. Invest 5, 175-187, and references to additional structures cited within.

48. Jenne, D. E., Denzel, K., Blatzinger, P., Winter, P., Obermaier, B., Linke, R. P., and Altland, K. (1996) Proc. Natl. Acad. Sci. U.S.A. 93, 6302-6307.

49. Sebastiao, P. M., Saraiva, M. J., and Damas, M. A. (1998) J. Biol. Chem. 38, 24715-24722.

50. Nettleton, E. J., Sunde, M., Lai, Z., Kelly, J. W., Dobson, C. M., and Robinson, C. V. (1998) J. Mol. Biol. 281, 553-564.

51. Colon, W., Lai, Z. McCutchen, L. S., Miroy, J. G., Strang, C. and Kelly, W. J. (1996) in The nature and origin of amyloid fibrils. Wiley, Chichester (Ciba Foundation Symposium 1996), $228-242$.

52. Quintas, A., Saraiva, M. J. M., and Brito, R. M. M. (1997) FEBS Lett. 418, 297-300.

53. Goldsteins, G., Anderson, A., Olofsson, A., Dacklin, I., Edvinsson, A., Baranov, V., Sandgren, O., Thylen, C., Hammarstrom, S., and Lundgren, E. (1997) Biochemistry 36, 5346-5352.

54. Ho, S. C., and Wemmer, D. (unpublished results).

55. Andreu, J. M., and Timasheff, S. N. (1986) Methods Enzymol. 130, 47-59.

56. Mulkerrin, M. G., and Wetzel, R. (1989) Biochemistry 28, 6556-6561.

57. Klunk, W. E., Pettegrew, J. W., and Abraham, D. J. (1989) J. Histochem. Cytochem. 37, 1273-81.

58. Glenner, G. G., Eanes, E. D., and Page, D. L. (1972) J. Biochem. Cytochem. 20, 821-826.

59. Philo, S. J. (1997) Biophys. J. 72, 435-444.

60. Demeler, B., Saber, H., and Hansen, J. C. (1997) Biophysical J. 72, 397-407.

61. van Holde, K. E., and Weischet, W. O. (1978) Biopolymers 17, 1387-1403.

62. Stafford, W. F. (1992) Anal. Biochem. 203, 295-301.

63. Cheng, S. Y., Pages, R. A., Saroff, H. A., Edelhoch, H., and Robbins, J. (1977) Biochemistry 16, 3707-3713.

64. Almeida, M., R. Saraiva, Maria J. (1996) Eur. J. Endocrinol., $226-230$. 
65. Nybo, M., Hackler, R., Kold, B., Nielsen, H., Steinmetz, A., and Svehag, S. E. (1998) Scand. J. Immunol. 48, 350-356.

66. Lai, Z., McCulloch, J., Lashuel, H. A., and Kelly, J. W. (1997) Biochemistry 36, 10230-10239.

67. Kuwajima, K. (1989) PROTEINS: Structure, Function and Genetics 6, 87-103.
68. Murakami, T., Ohnishi, S., Nishiguchi, S., Maeda, S., Araki, S., and Shimada, K. (1988) Biochem. Biophys. Res. Comm. $155,554-60$.

69. Kraulis, P. J. (1991) J. Appl. Cryst 24, 946-950.

BI991021C 